\title{
LISTADO PRELIMINAR DE MANTODEA, BLATTODEAY ORTHOPTERA (INSECTA) DE LA REGIÓN DE PIURA, PERÚ
}

\author{
Gino Juárez-Noé ${ }^{1, *}$ \& Uzbekia González-Coronado² \\ 'Distrito de Piura, Región de Piura-Perú. Email: norbiol@hotmail.com \\ ORCID iD: https://orcid.org/0000-0002-8847-6059 \\ 2Distrito de Piura, Región de Piura-Perú. Email: issa.gonzalez06@gmail.com \\ ORCID iD: https://orcid.org/0000-0001-5920-7549 \\ *Autor para correspondencia: Gino Juárez-Noé email: norbiol@hotmail.com
}

\section{RESUMEN}

Se ha elaborado un listado preliminar de mántidos, blatodeos y ortópteros de la región de Piura en Perú a partir de colectas de especímenes y registros en la bibliografía. En total se han reportado 32 especies de 15 familias, de las cuales cuatro especies pertenecen a Mantodea, cinco especies a Blattodea y 23 especies a Orthoptera. Se registra por primera vez para Perú a Chromacris psittacus psittacus (Gerstaecker, 1873). Se presentan los primeros registros de localidades precisas para el país de Thrasyderes leprosus Bolívar, 1881, mientras que Liturgusa maya Saussure \& Zehntner, 1894 es nuevo registro para la región Piura. En el caso de Musonia margharethae (Battiston \& Picciau, 2008), Pseudovates peruviana (Rehn, 1911) y Jivarus pictifrons Ronderos, 1979 amplían sus localizaciones dentro de la región. El $75 \%$ de las especies reportadas se debieron a colectas y el $25 \%$ a registros en la bibliografía. Todas las especies registradas se presentan a modo de listado faunístico comentado, incluyendo para cada una datos de colecta así como su distribución por provincia y paisaje ecológico.

Palabras clave: Lista faunística, distribución, paisaje ecológico, Mantodea, Blattodea, Orthoptera, Piura.

\section{ABSTRACT}

Preliminary checklist of the Mantodea, Blattodea and Orthoptera (Insecta) of Piura region, Peru

The first checklist of Mantodea, Blattodea and Orthoptera of Piura region in Peru is presented based on samplings of specimens and records in the bibliography. In total 32 species from 15 families have been reported of which four species belong to Mantodea, five species to Blattodea and 23 species to Orthoptera. Chromacris psittacus psittacus (Gerstaecker, 1873) is recorded for the first time from Peru. Exact locality records for Thrasyderes leprosus Bolívar, 1881 are given for the first time for Peru, while Liturgusa maya Saussure \& Zehntner, 1894 is a new record for Piura region. In the case of Musonia margharethae (Battiston \& Picciau, 2008), Pseudovates peruviana (Rehn, 1911) and Jivarus pictifrons Ronderos, 1979 expand their locations within the region. $75 \%$ of the recorded species were obtained by direct samplings and $25 \%$ previous records. We present a commented faunistic list that includes all the recorded species sampling data, distribution by province and ecological landscape.

Keywords: Checklist, distribution, ecological landscape, Mantodea, Blattodea, Orthoptera, Piura.

Cómo citar este artículo/Citation: Juárez-Noé, G. \& González-Coronado, U. 2020. Listado preliminar de Mantodea, Blattodea y Orthoptera (Insecta) de la región de Piura, Perú. Grael/sia, 76(1): e103. https://doi.org/10.3989/graellsia.2020. v76.240

Copyright: (C) 2020 SAM \& CSIC. This is an open-access article distributed under the terms of the Creative Commons Attribution 4.0 International (CC BY 4.0) License. 


\section{Introducción}

El super orden Dictyoptera está constituido por los órdenes Blattodea y Mantodea (Klass \& Meier, 2006; Beccaloni \& Eggleton, 2013). Blattodea, que comprende a las cucarachas y termitas, contiene cerca de 7570 especies conocidas (Beccaloni \& Eggleton, 2013), de las cuales aproximadamente 4600 especies son cucarachas y 2929 especies son termitas (Krishna et al., 2013; Beccaloni, 2014), mientras que Mantodea, que incluye a las mantis religiosas, está compuesto por aproximadamente 2450 especies con cerca de 498 especies en el neotrópico (Rivera, 2010; Otte et al., 2018). El orden Orthoptera, en los que se incluyen a saltamontes y grillos, comprende aproximadamente 28040 especies, distribuidas en 40 familias y 4418 géneros (Ingrisch, 2011; Cigliano et al., 2018). Perú no cuenta con una aproximación exacta de la cantidad de especies de Mantodea, Blattodea y Orthoptera que posee, sin embargo existen algunos trabajos que detallan la riqueza y distribución de las especies para algunos grupos de estos órdenes presentes en el país, tal es el caso de las familias Gryllidae con 81 especies (Aguilar, 1973), Proscopiidae con 22 especies (Aguilar, 1983), Acrididae con 114 especies (Beingolea-Guerrero, 1989) y Mantidae con 67 especies (Rivera \& Vergara-Cobián, 2017).

La gran variedad fisiográfica y climática que caracteriza a la región Piura, han determinado la existencia de zonas geomorfológicas y ecológicas importantes, originando una gran heterogeneidad de ecosistemas y variados gradientes altitudinales, lo que ha dado lugar a una notable riqueza vegetacional y faunística con altos niveles de endemismos (More et al., 2014). El objetivo de esta contribución es elaborar una lista preliminar de mántidos, blatodeos y ortópteros, presentes para la región de Piura, Perú, realizada a partir de colectas de especímenes y registros en la bibliografía.

\section{Material y métodos}

La Región de Piura se ubica en el extremo noroeste de Perú $\left(04^{\circ} 59^{\prime} \mathrm{S}-80^{\circ} 05^{\prime} \mathrm{O}\right)$, cuenta una superficie aproximada de $35892.49 \mathrm{~km}^{2}$ y está dividida en ocho provincias: Piura, Sechura, Sullana, Talara, Paita, Morropón, Huancabamba y Ayabaca (Fig. 1). Su clima es desértico y semidesértico en la costa con temperaturas máximas de $39^{\circ} \mathrm{C}$ y mínimas de $18^{\circ} \mathrm{C}$, y húmedo y frío en la sierra con máximas de $15^{\circ} \mathrm{C}$ y mínimas de $8^{\circ} \mathrm{C}$ siendo sus precipitaciones generalmente estacionales (diciembre a abril). Posee dos cadenas montañosas, las cuales, configuran el relieve, el paisaje y el contraste altitudinal de la región: la Cordillera de Los Amotapes (hasta los $1600 \mathrm{~m}$ de altitud) y la Cordillera de los Andes (hasta casi los $4000 \mathrm{~m}$ de altitud). Ecológicamente la región está conformado en su mayoría por bosques estacionalmente secos y desierto costero, y en menor proporción por manglares, bosques secos interandinos, matorral montano, bosques montanos de la vertiente occidental (bosques de neblina), páramo y meseta andina (More et al., 2014; MINAM, 2018).

Todas las especies consideradas en esta lista se basan en colectas de especímenes y registros en la bibliografía. Los registros a través de colectas se realizaron en 43 localidades de muestreo que abarcaron las ocho provincias de la región en un rango altitudinal desde el nivel del mar hasta los $3100 \mathrm{~m}$ de altitud (Apéndice 1). Las colectas tuvieron una duración de dos días por localidad y se llevaron a cabo mensualmente desde el 2015 hasta el 2018. En cada localidad de muestreo se realizó recolecta directa revisando principalmente hojas, tallos y flores de plantas, hojarasca, suelo, troncos podridos y bajo piedras (Márquez-Luna, 2005), así como barridos sobre la vegetación (seis pases de red) en transectos de longitud no definida utilizando una red entomológica formada por vara rígida de $1.20 \mathrm{~m}$ de largo unida a un anillo metálico de $30 \mathrm{~cm}$ de diámetro y un tul de forma cónica de $90 \mathrm{~cm}$ de largo. Del mismo modo, se utilizó una trampa luz de dos focos de 100 watts cada uno, de luces blancas, colocadas frente y debajo de mantas blancas de dos metros de ancho y dos metros de largo, las cuales estuvieron activas entre las 19:00 h y 23:00 h.

Los registros bibliográficos provienen de las publicadas en revistas científicas, quedando excluidos los informes técnicos o de impacto ambiental y resúmenes de congresos. Los trabajos consultados fueron los de Aguilar (1973), Radclyffe (1979), Cigliano \& Amedegnato (2010) y Rivera \& Vergara-Cobián (2017).

Las identificaciones de los especímenes colectados fueron hechas consultando los trabajos de Caudell (1912), Rehn (1944), Carbonell \& Descamps (1978), Otte (1979), Mizukubo (1981), Radclyffe \& Carbonell (1982), Rivera (2001), Song (2009), Cigliano \& Amedegnato (2010), Svenson (2014), Cadena-Castañeda \& Cardona-Granda (2015), CadenaCastañeda (2015). Algunos de los ejemplares colectados fueron depositados en la colección del Museo de Entomología de la Universidad Nacional de Tumbes, Perú (MEUNT).

Todas las especies registradas se ordenaron siguiendo la clasificación de Ingrisch (2011) para Orthoptera, Beccaloni \& Eggleton (2013) para Blattodea y Rivera \& Vergara-Cobián (2017) para Mantodea y se presentan a modo de listado faunístico comentado, siendo la secuencia del material estudiado la siguiente: número de ejemplares ( $\widehat{\jmath},+q+$ ), localidad (país, provincia, distrito, localidad), coordenadas geográficas, altitud, fecha de captura, colector, método de captura, microhábitats, además de una referencia para indicar los autores de los trabajos revisados cuando se recopiló a través de bibliografía. Asimismo, para cada especie se registra su distribución por provincia: Piura (PIU), 


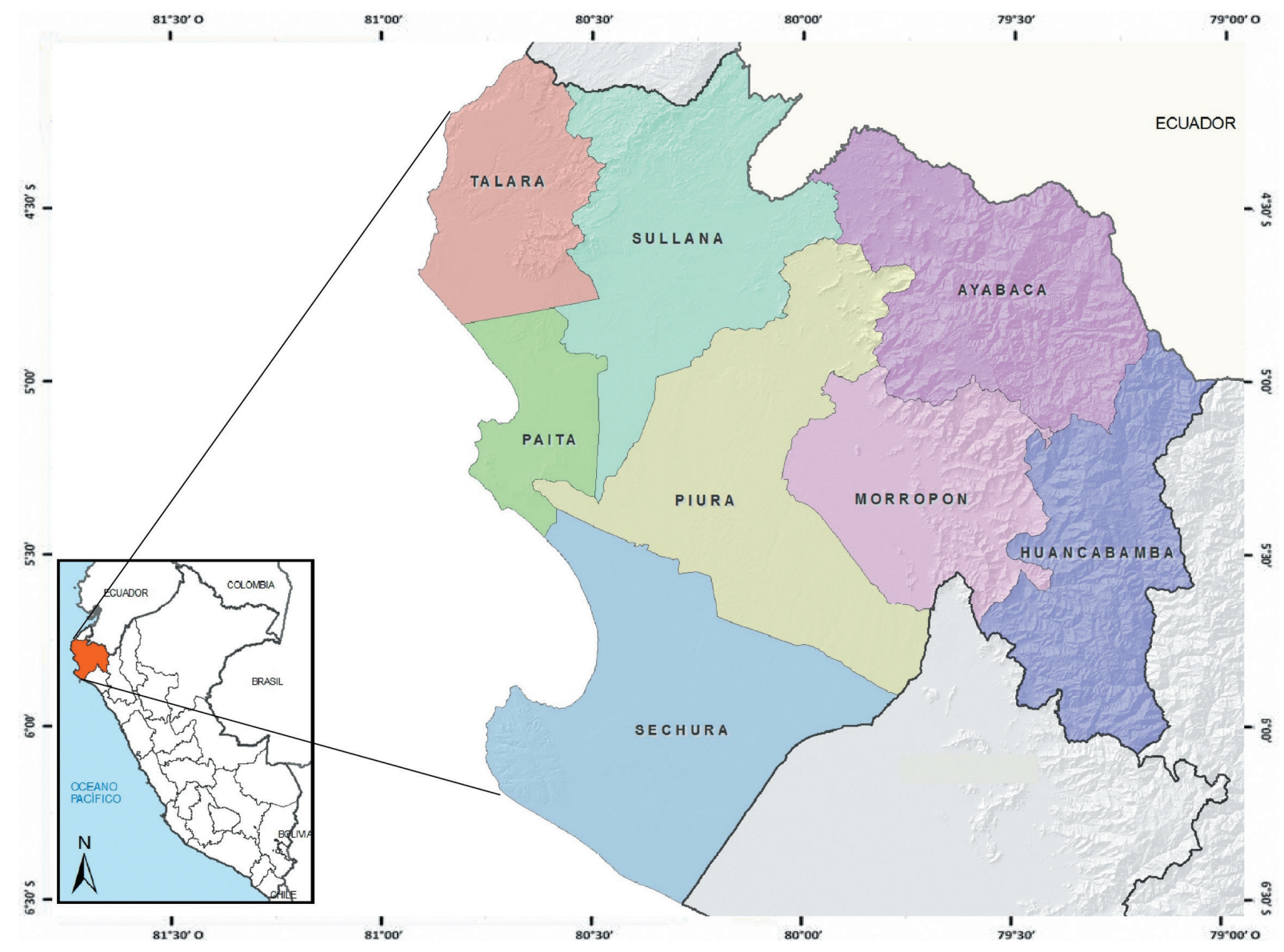

Fig. 1.- Mapa de localización de la región Piura.

Fig. 1.- Location map of the Piura region.

Sullana (SULL), Sechura (SECH), Paita (PAI), Talara (TAL), Morropón (MORR), Huancabamba (HUA) y/o Ayabaca (AYA) y por paisaje ecológico (More et al., 2014): Manglar (MAN) y Desierto Costero (DES) ambos a nivel del mar, Bosques Estacionalmente Secos de llanura (BESll, de 0 a $500 \mathrm{~m}$ ), Bosques Estacionalmente Secos de colina (BESc, de $500 \mathrm{~m}$ a $1000 \mathrm{~m}$ ), Bosques Estacionalmente Secos de montaña (BESm, de $1000 \mathrm{~m}$ a $1500 \mathrm{~m}$ ), Bosques Secos Interandinos (BSI, de $1500 \mathrm{~m}$ a $2000 \mathrm{~m}$ ) y Bosques Húmedos de Montaña (BHM, de 2000 m a 3100 m). Se resaltan en negrita las provincias en las especies que presentan ampliaciones de distribución.

\section{Resultados y discusión}

En total se han registrado 32 especies agrupadas en 31 géneros y 15 familias. De este total, cuatro especies correspondieron a Mantodea, cinco especies (incluyendo una sin identificar) correspondieron a Blattodea y 23 especies (incluyendo nueve sin identificar) correspondieron a Orthoptera. Las familias Acrididae y Tettigoniidae (Orthoptera) con ocho cada una, Thespidae (Mantodea) y Blaberidae (Blattodea) con dos cada una y el género Jivarus Giglio-Tos, 1898 con dos presentaron mayor cantidad de especies. La cantidad de especies registrada no es comparable con las 317 especies del orden Coleoptera (Juárez-Noé \& González-Coronado, 2019) y 140 especies del orden Hymenoptera (Juárez-Noé \& González-Coronado, 2018) citadas para la misma región, grupos taxonómicos mucho más diversos a nivel mundial y nacional (Bouchard et al., 2011; Chaboo, 2015; Fernández y Pujade-Villar, 2015).

Como producto del trabajo de colectas se logró obtener el $75 \%$ (24 especies) del total de las especies registradas, mientras que el $25 \%$ restante ( 8 especies) se logró a través de registros bibliográficos. Asimismo, del total de especies registradas a través de registros bibliográficos, solo Musonia margharethae (Battiston \& Picciau, 2008), Pseudovates peruviana (Rehn, 1911) y Jivarus pictifrons Ronderos, 1979 fueron recolectadas nuevamente, lo que permitió confirmar los datos y registros provenientes de estas fuentes, siendo las especies no recolectadas Pseudopogonogaster kanjaris Rivera \& Yagui, 2011, Jivarus discoloris Cigliano \& Amedegnato, 2010, Stenacris fissicauda chocoensis 
(Hebard 1923), Gryllus (Gryllus) capitatus Saussure, 1874 y Ornebius peruviensis Chopard, 1913. Es importante mencionar que las colectas de algunas especies resultaron ser nuevos registros, tal es el caso de Chromacris psittacus psittacus (Gerstaecker, 1873) que se cita por primera vez para Perú, de Liturgusa maya Saussure \& Zehntner, 1894 que se cita por primera vez para la región Piura, de $M$. margharethae, $P$. peruviana y $J$. pictifrons que amplían sus localizaciones dentro de la región y de Thrasyderes leprosus Bolívar, 1881 de la cual se entregan los primeros registros de localidades exactas en el país. Todo lo anterior demuestra la gran importancia que tienen las colectas entomológicas en lugares poco explorados, como es el caso de la región Piura, ya que la mayoría de las exploraciones entomológicas en Perú se han centrado mayormente en regiones netamente andinas y selváticas (Chaboo, 2015).

Aunque no se analizó el grado de eficiencia de los métodos de colecta, es importante mencionar que para la mayoría de los individuos de $M$. margharethae, P. peruviana, Schistocerca interrita Scudder, 1889, Trimerotropis ochraceipennis reedi Liebermann, 1945, J. pictifrons, Orphulella punctata (De Geer, 1773), Anchotatus brevicornis (Caudell, 1912), T. leprosus, C. psittacus psittacus (Gerstaecker, 1873), Moncheca sp. no identificada 1, Conocephalus sp. no identificada 1, Neoconocephalus sp. no identificada 1, Typophyllum sp. no identificada 1 y Stilpnochlora sp. no identificada 1 fue indispensable el uso de red entomológica como método de captura, ya que estas especies se encontraron preferentemente entre la vegetación arbórea, arbustiva y herbácea incluso en áreas despejadas de bosques desprovistas de vegetación. Del mismo modo, la colecta manual fue indispensable principalmente para la captura de blatodeos y termitas ya que se colectaron bajo la hojarasca y debajo de piedras, como Pycnoscelus surinamensis (Linnaeus, 1758), Blaberus sp. no identificada 1 y Neoscapteriscus sp. no identificada 1, en el interior de ramas y troncos de árboles secos y podridos, como Incisitermes immigrans (Snyder, 1922) y deambulando en el interior de domicilios, como Periplaneta americana Linnaeus, 1758, Blattella germanica Caudell, 1903 y P. surinamensis. Aunque la mayoría de insectos son atraídos por la luz, solo Ceraia sp. no identificada 1, Scudderia sp. no identificada 1 y Ellipes occidentalis Günther, 1977 fueron capturados mediante trampas luz, estas mismas especies también fueron colectadas simultáneamente a través de colecta manual y red entomológica.

La fauna de mántidos, blatodeos y ortópteros de la región Piura presenta una mezcla de especies de zonas secas-desérticas y andinas, que podría deberse a la gran diversidad de tipos de vegetación y paisajes ecológicos que presenta esta región, hechos que influyen en la distribución general de las especies (More et al., 2014). Así, algunas especies mostraron distribución concordante con los tipos de paisaje ecológico evaluados, tal es el caso para $M$. margharethae, P. peruviana, L. maya, T. ochraceipennis reedi, $S$. interrita, A. brevicornis y I. immigrans, cuya distribución se da en bosques estacionalmente secos y desierto costero (Rivera, 2001; Svenson, 2014; Rivera \& Vergara-Cobián, 2017) y para P. kanjaris, Abracris flavolineata (De Geer, 1773), Nepiopteracris eburnifrons (Bruner, 1910) , J. pictifrons, J. discoloris, Moncheca sp. no identificada 1 y Typophyllum sp. no identificada 1 , cuya distribución se da a partir de bosques secos interandinos y bosques húmedos de montaña (Cigliano \& Amedegnato, 2010; Rivera \& Vergara-Cobián, 2017). Sin embargo, algunas especies mostraron amplia distribución abarcando todas las provincias y paisajes ecológicos, esto específicamente para los ortópteros Conocephalus sp. no identificada 1 y Neoconocephalus sp. no identificada 1 y los blatodeos $P$. americana, B. germanica, Blaberus sp. no identificada 1 y $P$. surinamensis (Linnaeus, 1758) ya que se adaptan perfectamente a zonas húmedas, secas y desérticas tanto en bosques como en el interior de domicilios (Torres, 2015).

Es importante mencionar, que algunas de las especies registradas revisten de un gran interés económico ya que poseen la capacidad de constituirse como plagas en la agricultura tal es el caso de $S$. interrita, Conocephalus sp. no identificada 1, Neoconocephalus sp. no identificada 1 y Neoscapteriscus sp. no identificada 1, que han causado daños en diversos cultivos de la costa norte de Perú (Beingolea, 1985; Pisfil-Llontop, 1985), mientras que $P$. americana, B. germanica, $P$. surinamensis, Blaberus sp. no identificada 1 tienen interés por ser plagas domesticas habitando en viviendas y establecimientos humanos causando molestias y perdida de material, así como por ser posibles vectores de enfermedades (Ramírez-Pérez, 1989; Torres, 2015) y de I. immigrans por ser considerada como plaga en la industria maderera (Scheffrahn, 1994).

Por último, es necesario completar colectas en localidades aún no exploradas, especialmente en las partes andinas más altas por encima de los $3200 \mathrm{~m}$ de altitud, donde se suceden ecosistemas de páramos y mesetas andinas (More et al., 2014), con lo que probablemente se eleve el número de especies de mántidos, blatodeos y ortópteros.

\section{Listado Faunístico}

ORDEN BLATTODEA Brunner von Wattenwyl, 1882

Familia Ectobiidae Brunner von Wattenwyl, 1865

Subfamilia Blattellinae, Karny 1908

Género Blattella Caudell, 1903

\section{Blattella germanica Linnaeus, 1767}

Material eXAminado. 1 \%, Perú, Piura, Castilla, Universidad Nacional de Piura, $05^{\circ} 10^{\prime} 51^{\prime \prime} \mathrm{S}-80^{\circ} 37^{\prime} 31^{\prime \prime} \mathrm{O}, 29 \mathrm{msnm}$, 2-II-2018, U. González leg., colecta manual, Bosque estacionalmente seco de llanura; 1 , Sullana, Salitral, 0451'27"S-8040'52"O, 23 msnm, 6-IV-2017, G. Juárez leg., colecta manual, Bosque estacionalmente seco de 
llanura; 2 ふึ ô, Talara, Lobitos, $04^{\circ} 27^{\prime} 10^{\prime \prime} \mathrm{S}-81^{\circ} 16^{\prime} 40^{\prime \prime} \mathrm{O}$, 12 msnm, 27-VI-2015, G. Juárez leg., colecta manual, Desierto; 1 o , Paita, $05^{\circ} 04^{\prime} \mathrm{S}-81^{\circ} 06^{\prime} \mathrm{O}, 10 \mathrm{msnm}, 7-\mathrm{IX}$ 2015, G. Juárez leg., colecta manual, Bosque estacionalmente seco de llanura; $3 \hat{\jmath} \widehat{\jmath}$, Paita, Vichayal, 04 $51^{\prime} 55^{\prime \prime} \mathrm{S}-81^{\circ} 04^{\prime} 19^{\prime \prime} \mathrm{O}, 10 \mathrm{msnm}, 20-\mathrm{X}-2018$, Gino Juárez leg., colecta manual, Bosque estacionalmente

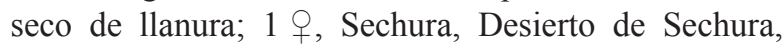
05³4'S-81¹6'O, 10 msnm, 13-XII-2016, G. Juárez leg., colecta manual, Desierto; 1 ㅇ, Morropón, Piedra del Toro, $05^{\circ} 11^{\prime} \mathrm{S}-80^{\circ} 49^{\prime} \mathrm{O}, 1225$ msnm, 29-VII-2016, G. Juárez leg., colecta manual, Bosque estacionalmente seco de colina; $1 \hat{\jmath}$, Huancabamba, Sondorillo, 05²7'07"S-79³6'22"O, 1600 msnm, 17-XI-2017, G. Juárez leg., colecta manual, Bosque seco interandino; 2 우, Huancabamba, Canchaque, Cerro Huayanay, $05^{\circ} 24^{\prime} 07^{\prime \prime} \mathrm{S}-79^{\circ} 36^{\prime} 22^{\prime \prime} \mathrm{O}, 1600 \mathrm{msnm}, 15-\mathrm{V}-2018$, U. González leg., colecta manual, Bosque

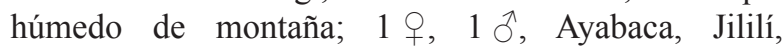

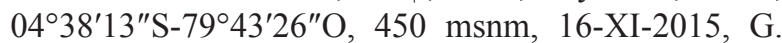
Juárez leg., colecta manual, Bosque seco interandino.

Distribución (Provincia) (paisaje ecológico). (PIU, SULL, TAL, PAI, SECH, MORR, AYA, HUA) (BES, BSI, DES, BHM).

Comentarios. Especie de distribución mundial considerada como plaga doméstica, además de tener importancia médica pues trasmite innumerables organismos patógenos como virus, bacterias, hongos, helmintos y responsable de enfermedades alérgicas como asma bronquial y rinitis alérgica (Iannacone \& Alvariño, 2007). Todos los individuos colectados fueron encontrados en ambientes urbanos (intradomiciliarios) en contacto con material orgánico en descomposición y en comedores y cocinas de viviendas tanto de día como de noche.

Familia Blaberidae Saussure, 1864

Subfamilia Blaberinae Saussure, 1864

Género Blaberus Serville, 1831

2. Blaberus sin identificar sp. 1 (Fig. 2)

Material eXAminado. 1 \% , Perú, Piura, Castilla, Universidad Nacional de Piura, $05^{\circ} 10^{\prime} 51^{\prime \prime} \mathrm{S}-80^{\circ} 37^{\prime} 31^{\prime \prime} \mathrm{O}, \quad 29$ msnm, 2-II-2015, colecta manual, U. González, Bosque estacionalmente seco de llanura; 1 \%, Sullana, Salitral, $04^{\circ} 51^{\prime} 27^{\prime \prime S}-80^{\circ} 40^{\prime} 52^{\prime \prime} \mathrm{O}, 23 \mathrm{msnm}, 6$-IV2016, colecta manual, G. Juárez, Bosque estacionalmente seco de llanura; 1 , Sullana, Querecotillo, 04 $50^{\prime} 24^{\prime \prime} \mathrm{S}-80^{\circ} 38^{\prime} 57^{\prime \prime} \mathrm{O}, 23 \mathrm{msnm}, 12-\mathrm{VI}-2016$, colecta manual, G. Juárez, Bosque estacionalmente seco de llanura; 1 o, Talara, Lobitos, $04^{\circ} 27^{\prime} 10^{\prime \prime} \mathrm{S}-81^{\circ} 16^{\prime} 40^{\prime \prime} \mathrm{O}, 12$ msnm, 27-VI-2015, colecta manual, G. Juárez, Desierto; 1 ․, Paita, $05^{\circ} 04^{\prime} \mathrm{S}-81^{\circ} 06^{\prime} \mathrm{O}, 10 \mathrm{msnm}, 7-\mathrm{IX}-2016$, colecta manual, G. Juárez, Bosque estacionalmente seco de llanura; 1 \&, Sechura, Desierto de Sechura, $05^{\circ} 34^{\prime} \mathrm{S}-81^{\circ} 16^{\prime} \mathrm{O}, 10 \mathrm{msnm}, 13-\mathrm{XII}-2017$, colecta manual, G. Juárez, Desierto; 1 \&, Morropón, Piedra del Toro, $05^{\circ} 11^{\prime} \mathrm{S}-80^{\circ} 49^{\prime} \mathrm{O}, 1225 \mathrm{msnm}, 29-\mathrm{VII}-2017$, colecta manual, G. Juárez, Bosque estacionalmente seco de colina; $1 \hat{\jmath}$, Huancabamba, Huancabamba,
05²7'07"S-79³6'22"O, 2600 msnm, 17-XI-2018, G. Juárez, Bosque húmedo de montaña; 1 \&, 1 స, Ayabaca, 0430'45"S-8002'10"O, 450 msnm, 15-XI-2017, colecta manual, G. Juárez, Bosque estacionalmente seco de colina.

Distribución (Provincia) (paisaje ecológico). (PIU, SULL, TAL, PAI, SECH, MORR, AYA, HUA) (BES, BSI, DES, BHM).

Comentarios. La mayoría de los individuos colectados se encontraron en ambientes forestales específicamente en troncos huecos de árboles secos en descomposición y entre la hojarasca, aunque también se colectaron en ambientes urbanos peridomiciliarios como jardines e intradomiciliarios especialmente en la cocina.

Subfamilia Pycnoscelinae

Género Pycnoscelus Scudder, 1862

3. Pycnoscelus surinamensis (Linnaeus, 1758)

Material eXAminado. 1 q, Perú, Piura, Castilla, 0510'51"S-80³7'31"O, 29 msnm, 12-IV-2016, U. González leg., colecta manual, Bosque estacionalmente seco de llanura; 2 ㅇ, Sullana, Salitral, 04 51'27"S-8040'52"O, 23 msnm, 25-VII-2017, G. Juárez leg., colecta manual, Bosque estacionalmente seco de llanura; 1 q, Talara, Los Órganos, 0410'38"S-81 07'29"O, 12 msnm, 11-IX-2018, G. Juárez leg., colecta manual, Desierto; 1 q, Paita, 0504'S-81 $06^{\prime} \mathrm{O}, 10$ msnm, 17-X-2018, G. Juárez leg., colecta manual, Bosque estacionalmente seco de llanura; 1 ㅇ, Sechura, Vice, $05^{\circ} 25^{\prime} \mathrm{S}-81^{\circ} 46^{\prime} \mathrm{O}$, 10 msnm, 06-XII-2017, U. González leg., colecta manual, Desierto; 2 우, Morropón, Santo Domingo, 0501'S-7952'O, 2110 msnm, 13-XI-2015, G. Juárez leg., colecta manual, Bosque húmedo de montaña; 1 స, Huancabamba, Huancabamba, 05²7'07"S-79³6'22"O, 2600 msnm, 22-VI-2015, G. Juárez leg., colecta manual, Bosque húmedo de montaña; 1 त, Ayabaca, Yacupampa, 043' $45^{\prime \prime S}-79^{\circ} 42^{\prime} 10^{\prime \prime} \mathrm{O}, 2750$ msnm, 15-XI-2017, G. Juárez, colecta manual, Bosque húmedo de montaña; 1 q, Ayabaca, Jililí, 04³8'13"S-7943'26"O, 450 msnm, 29-I-2016, U. González leg., colecta manual Bosque seco interandino.

Distribución (Provincia) (paisaje ecológico). (PIU, SULL, TAL, PAI, SECH, MORR, AYA, HUA) (BES, BSI, DES, BHM).

Comentarios. Especie nativa de la región Indomalaya, sin embargo ha sido introducida a países tropicales y subtropicales en todo el mundo incluyendo el continente americano, África, Australia e islas del Pacífico. En el continente americano ha sido introducida accidentalmente desde Estados Unidos hasta Argentina (Roth, 1998; Beccaloni, 2014). La mayoría de los individuos colectados fueron registrados en ambientes forestales ya sea en tierra suelta, debajo de piedras y sotobosque, aunque también se colectaron en ambientes urbanos peridomiciliarios como jardines e intradomiciliarios especialmente en baños y cocina, coincidiendo con los tipos de hábitat para esta especie (Pellens \& Grandcolas, 2002; Taucare-Ríos et al., 2018). 
Familia Blattidae Latreille, 1810

Subfamilia Blattinae Latreille, 1810

Género Periplaneta Burmeister, 1838

4. Periplaneta americana Linnaeus, 1758 (Fig. 3)

Material examinado. 3 q, Perú, Piura, Catacaos, $05^{\circ} 15^{\prime} 42^{\prime \prime} \mathrm{S}-80^{\circ} 40^{\prime} 27^{\prime \prime} \mathrm{O}, 29 \mathrm{msnm}, 16-\mathrm{VII}-2016$, U. González leg., colecta manual, Bosque estacionalmente seco de llanura; 3 우, $2 \bigcirc^{\Uparrow}$, Piura, Universidad de Piura, $05^{\circ} 10^{\prime} 11^{\prime \prime} \mathrm{S}-80^{\circ} 36^{\prime} 51^{\prime \prime} \mathrm{O}, 29 \mathrm{msnm}$, 27-IX-2018, U. González leg., colecta manual, Bosque estacionalmente seco de llanura; $2 \circ \circ$, Sullana, Salitral, 0451'27"S-8040'52"O, 23 msnm, 18-I-2016, G. Juárez leg., colecta manual, Bosque estacionalmente seco de llanura; 2 우, Sullana, Lancones, 043'ㄱ' $27^{\prime \prime} \mathrm{S}-80^{\circ} 32^{\prime} 55^{\prime \prime} \mathrm{O}$, 190 msnm, 14-XI-2015, G. Juárez leg., colecta manual, Bosque estacionalmente seco de colina; 1 , , Talara, Los Órganos, $04^{\circ} 10^{\prime} 38^{\prime \prime} \mathrm{S}-81^{\circ} 07^{\prime} 27^{\prime \prime} \mathrm{O}, 12 \mathrm{msnm}$, 05-V-2017, G. Juárez leg., colecta manual, Desierto; 1 ㅇ, Talara, El Alto, $04^{\circ} 16^{\prime} 04^{\prime \prime} \mathrm{S}-81^{\circ} 13^{\prime} 09^{\prime \prime} \mathrm{O}, 300$ msnm, 13-X-2017, G. Juárez leg., colecta manual, Bosque estacionalmente seco de colina; 1 q, Paita, $05^{\circ} 04^{\prime} \mathrm{S}-81^{\circ} 06^{\prime} \mathrm{O}, 10 \mathrm{msnm}, 21-\mathrm{II}-2017$, G. Juárez leg., colecta manual, Bosque estacionalmente seco de llanura; 1 q, Sechura, Vice, $05^{\circ} 25^{\prime} \mathrm{S}-81^{\circ} 46^{\prime} \mathrm{O}, 10$ msnm, 12-XII-2017, U. González leg., colecta manual, Desierto; 1 ô, Sechura, Sechura, $05^{\circ} 34^{\prime} \mathrm{S}-81^{\circ} 16^{\prime} \mathrm{O}, 16$ msnm, 24-VI-2017, U. González leg., colecta manual, Bosque estacionalmente seco de llanura; 1 + , Morropón, Chulucanas, $05^{\circ} 05^{\prime} \mathrm{S}-80^{\circ} 99^{\prime} \mathrm{O}, 350 \mathrm{msnm}, 22-\mathrm{I}-2015$, G. Juárez leg., colecta manual, Bosque estacionalmente seco de colina; 2 우, Morropón, Morropón, $05^{\circ} 05^{\prime} 51^{\prime \prime} \mathrm{S}-80^{\circ} 09^{\prime} 44^{\prime \prime} \mathrm{O}, \quad 557 \mathrm{msnm}, \quad 23-\mathrm{I}-2015$, G. Juárez leg., colecta manual, Bosque estacionalmente

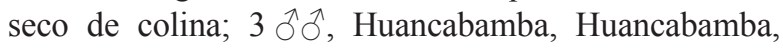
$05^{\circ} 27^{\prime} 07^{\prime \prime S}-79^{\circ} 36^{\prime} 22^{\prime \prime} \mathrm{O}, 2567$ msnm, 15-VI-2018, G. Juárez leg., colecta manual, Bosque húmedo de montaña;

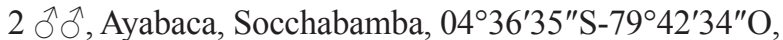
2755 msnm, 26-VII-2015, G. Juárez leg., colecta manual, Bosque húmedo de montaña; 1 \%, Ayabaca, Jililí, 04³8'13"S-7943'26"O, 450 msnm, 29-I-2016, U. González leg., colecta manual, Bosque seco interandino.

Distribución (Provincia) (paisaje ecológico). (PIU, SULL, TAL, PAI, SECH, MORR, AYA, HUA) (BES, BSI, DES, BHM).

Comentarios. Especie cosmopolita de origen africano considerada plaga doméstica e importante vector mecánico y transmisor de microorganismos que afectan la salud del hombre (Schapheer et al., 2018). Fernández et al., (2001) realizaron en Perú un estudio sobre los parasitos presentes en esta especie encontrando cuatro parasitos propios de la fauna natural: Lophomonas blattarum Stein, 1860 (Mastigophora, Lophomonadidae), Leptomonas sp (Mastigophora, Trypanosomatidae), Hammerschdmidtiella diesigni (Hammerschmidt, 1838), Leydinema appendiculatum (Leidy, 1850) (Nematoda, Thelastomatidae) y siete parásitos que pueden ser transmitidos al hombre: Giardia lamblia Kofoid \& Christiansen, 1915 (Mastigophora,
Hexamitidae), Endolimax nana (Wenyon \& O'connor, 1917) Entamoeba coli (Grassi, 1879), Iodamoeba butschilii (Prowazek, 1911) (Sarcodina, Entamoebidae), Chilomastix mesnili (Wenyon, 1910) (Mastigophora, Retortamonidae), Blastocystis hominis Brumpt, 1912 (Sarcodina, Blastocystida), Cryptospordium sp (Coccidia, Cryptosporidiidae). Todos los individuos colectados fueron encontrados en ambientes urbanos (peridomiciliarios e intradomiciliarios) en contacto con material orgánico en descomposición, sistemas de alcantarillado y en comedores y cocinas de viviendas tanto de día como de noche.

Familia Kalotermitidae Froggatt, 1896

Género Incisitermes Froggatt, 1906

5. Incisitermes immigrans (Snyder, 1922) (Fig. 4)

Material examinado. 2 우, Perú, Talara, Los Órganos, 0410'38"S-81 07'27"O, 12 msnm, 15-VI-2016, G. Juárez leg., colecta manual, Desierto; $1 \hat{\jmath}$, Talara, El Alto, 04 $16^{\prime} 04^{\prime \prime} \mathrm{S}-81^{\circ} 13^{\prime} 09^{\prime \prime} \mathrm{O}, 300 \mathrm{msnm}, 23-\mathrm{IX}-2016$, G. Juárez leg., colecta manual, Bosque estacionalmente seco de colina.

Distribución (Provincia) (paisaje ecológico). (TAL) (BES, DES).

Comentarios. Las especies del genero Incisitermes se distribuyen en la región neártica, neotropical, Australia, India e Islas del Océano Pacífico, y al igual que la mayoría de especies de termitas es considerada plaga común de la industria maderera y de especies forestales (Scheffrahn, 1994). Los individuos colectados fueron encontrados en el interior de troncos secos muertos de Prosopis pallida (Humb. \& Bonpl.ex.Wild.).

\section{ORDEN MANTODEA}

Familia Thespidae Saussure, 1869

Subfamilia Pseudopogonogastrinae Rivera \& Svenson, 2016

Género Pseudopogonogaster Beier, 1942

6. Pseudopogonogaster kanjaris Rivera \& Yagui, 2011

Material examinado. 1 $\widehat{\jmath}$, Perú, Ayabaca, Bosque Los Molinos, 04³7'30"S-7944'36"O, 2494 msnm, 18-VIII-2009, D. Chunga \& O. Huaches leg., Bosque Húmedo de Montaña.

Distribución (Provincia) (paisaje ecológico). (AYA) (BHM).

Comentarios. Las especies del género Pseudopogonogaster se distribuyen a lo largo de los Andes tropicales de Colombia, Ecuador y norte de Perú (Rivera et al., 2011). En Perú, $P$. kanjaris se distribuye en bosques húmedos de montaña de las regiones de Piura y Lambayeque (Rivera et al., 2011; Rivera \& Vergara-Cobián, 2017).

REFERENCIA. Rivera \& Vergara-Cobián (2017).

Subfamilia Thespinae Saussure, 1869

Género Musonia Stål, 1877

7. Musonia margharethae (Battiston \& Picciau, 2008) (Fig. 5)

Material examinado. 1 $\lesssim$, Piura, 25-VII-1968, O. Velarde leg.; 1 \&, Piura, Castilla, Universidad Nacional de 
Piura, $05^{\circ} 10^{\prime} 51^{\prime \prime} \mathrm{S}-80^{\circ} 37^{\prime} 31^{\prime \prime} \mathrm{O}, 29 \mathrm{msnm}, 2-\mathrm{II}-2018$, U. González leg., red entomológica, Bosque estacionalmente seco de llanura; 19 , Piura, Universidad de Piura, $05^{\circ} 10^{\prime} 11^{\prime \prime} \mathrm{S}-80^{\circ} 36^{\prime} 51^{\prime \prime} \mathrm{O}, 29 \mathrm{msnm}, 29-\mathrm{VI}-$ 2017, G. Juárez leg., red entomológica, Bosque estacionalmente seco de llanura; 1 , Sullana, Salitral, 0451'27"S-8040'52"O, 23 msnm, 09-XII-2015, G. Juárez leg., red entomológica, Bosque estacionalmente seco de llanura; 1 , Talara, Los Órganos, 0410'38"S-81 07'27"O, 12 msnm, 17-VII-2016, G. Juárez leg., colecta manual, Bosque estacionalmente seco de llanura; 1 ․ Talara, El Alto, 04 ${ }^{\circ} 16^{\prime} 04^{\prime \prime} \mathrm{S}-81^{\circ} 13^{\prime} 09^{\prime \prime} \mathrm{O}$, 8 msnm, 21-IX-2017, G. Juárez leg., red entomológica, Desierto; 1 q, Paita, $05^{\circ} 04^{\prime} \mathrm{S}-81^{\circ} 06^{\prime} \mathrm{O}, 10 \mathrm{msnm}$, 02-VIII-2017, G. Juárez leg., red entomológica, Bosque estacionalmente seco de llanura; 1 q, Sechura, Vice, 05²5'S-8146'O, 10 msnm, 23-X-2017, U. González leg., red entomológica, Desierto; 1 đ̄, Sechura, Sechura, $05^{\circ} 34^{\prime} \mathrm{S}-81^{\circ} 16^{\prime} \mathrm{O}, 16$ msnm, 24-VI-2017, U. González leg., red entomológica, Bosque estacionalmente seco de llanura; 1 + , Morropón, Chulucanas, $05^{\circ} 05^{\prime} \mathrm{S}-80^{\circ} 99^{\prime} \mathrm{O}$, 350 msnm, 13-VIII-2018, G. Juárez leg., red entomológica, Bosque estacionalmente seco de colina; 2 우, Morropón, Morropón, $05^{\circ} 05^{\prime} 51^{\prime \prime S}-80^{\circ} 09^{\prime} 44^{\prime \prime} \mathrm{O}, 557$ msnm, 23-I-2015, G. Juárez leg., red entomológica, Bosque estacionalmente seco de colina.

Distribución (Provincia) (paisaje ecológico). (PIU, SULL, TAL, PAI, SECH, MORR) (BES, DES).

Comentarios. Especie que se distribuye en ambientes semiáridos del sur de América Central y de América del Sur a lo largo de las zonas bajas de la costa del Pacífico (Rivera \& Vergara-Cobián, 2017). La especie amplia su localización dentro de la región a las provincias de Sullana, Paita, Talara, Sechura y Morropón, anteriormente solo se localizaba en la provincia de Piura (Rivera \& Vergara-Cobián, 2017). Los individuos colectados fueron encontrados sobre las hojas de vegetación arbórea, arbustiva y herbácea en ambientes áridos y semiáridos coincidiendo con el tipo de hábitat para esta especie. REFERENCIA. Rivera \& Vergara-Cobián (2017).

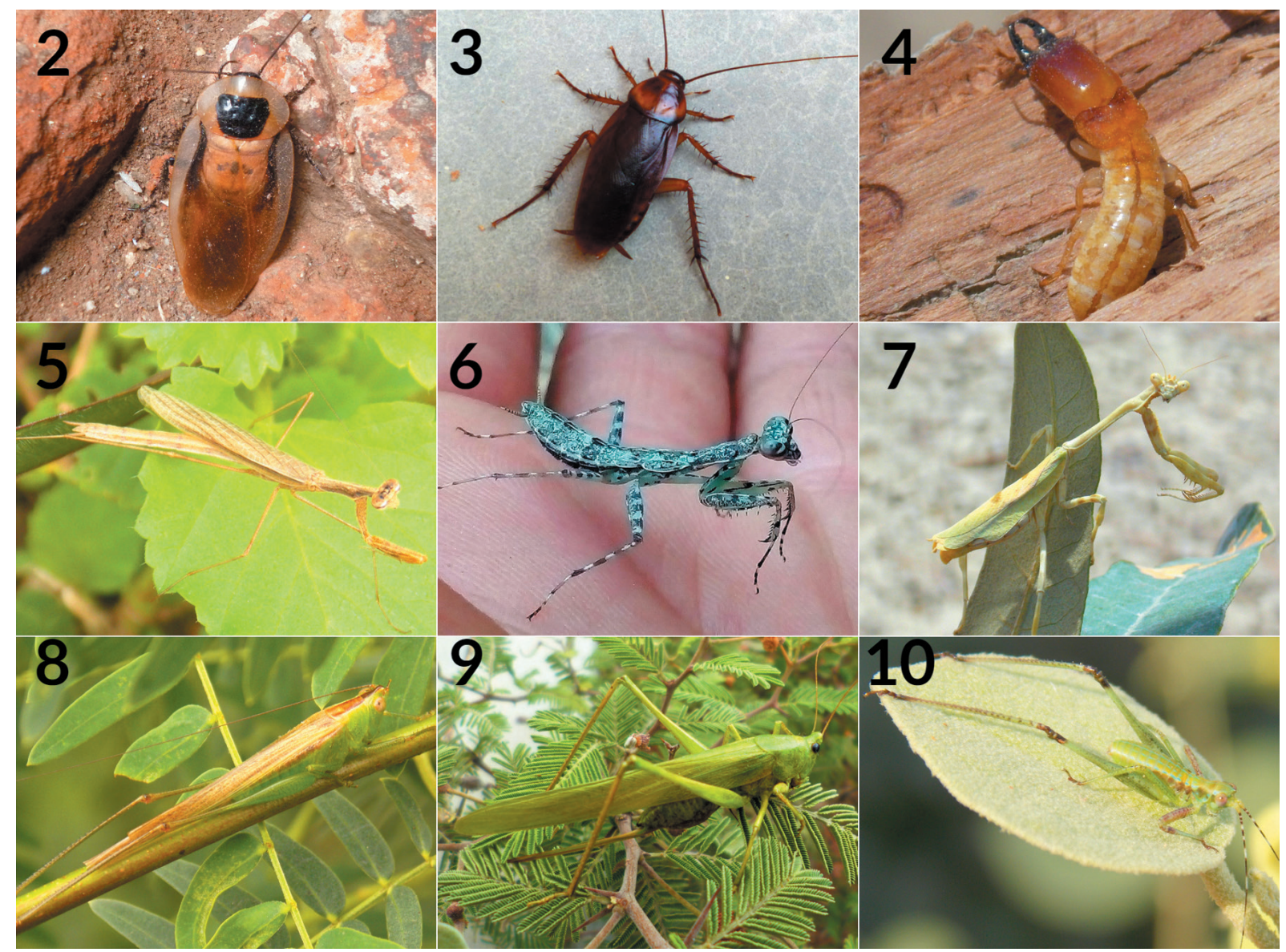

Figs. 2-10.- Habitus. 2. Blaberus sin identificar sp. 1. 3. Periplaneta americana Linnaeus, 1758. 4. Incisitermes immigrans (Snyder, 1922). 5. Musonia margharethae (Battiston \& Picciau, 2008). 6. Liturgusa maya Saussure \& Zehntner, 1894. 7. Pseudovates peruviana (Rehn, 1911). 8. Conocephalus sin identificar sp. 1. 9. Neoconocephalus sin identificar sp. 1. 10. Scudderia sin identificar sp. 1 (ninfa). [Fotografías 2-5, 7-10 de G. Juárez y U. González; Fotografía 6 de C. Caballero].

Figs. 2-10.- Habitus. 2. Blaberus unidentified sp. 1. 3. Periplaneta americana Linnaeus, 1758. 4. Incisitermes immigrans (Snyder, 1922). 5. Musonia margharethae (Battiston \& Picciau, 2008). 6. Liturgusa maya Saussure \& Zehntner, 1894. 7. Pseudovates peruviana (Rehn, 1911). 8. Conocephalus unidentified sp. 1. 9. Neoconocephalus unidentified sp. 1. 10. Scudderia unidentified sp. 1 (ninfa). [Photographs 2-5, 7-10 by G. Juárez and U. González; Photographs 6 by C. Caballero]. 
Familia Liturgusidae Giglio-Tos, 1915

Género Liturgusa Saussure, 1869

8. Liturgusa maya Saussure \& Zehntner, 1894 (Fig. 6)

Material examinado. 1 q, Perú, Morropón, Morropón, 0505'51"S-8009'44"O, 557 msnm, 19-VI-2015, G. Juárez leg., red entomológica, Bosque estacionalmente seco de colina.

Distribución (Provincia) (paisaje ecológico). (MORR) (BES).

Comentarios. Esta especie se distribuye en bosques tropicales húmedos y bosques estacionalmente secos de México, Costa Rica, Panamá, Honduras, Nicaragua, Venezuela, Ecuador, Colombia y Perú (Svenson, 2014). Nuevo registro para la región Piura, anteriormente se registraba para las regiones de Cajamarca, Cusco, Junín, Madre de Dios, Ucayali y Tumbes (Rivera \& VergaraCobián, 2017). El individuo colectado se encontró entre la vegetación herbácea en bosque estacionalmente seco, coincidiendo con el tipo de hábitat para esta especie.

Familia Mantidae Latreille, 1802

Subfamilia Vatinae Stål, 1877

Género Pseudovates Saussure, 1869

9. Pseudovates peruviana (Rehn, 1911) (Fig. 7)

Material EXAMINAdo. 1 q, Perú, Piura, Piura, 05 $12^{\prime} 03^{\prime \prime} \mathrm{S}-80^{\circ} 37^{\prime} 31^{\prime \prime} \mathrm{O}, \quad 29 \mathrm{msnm}, \quad$ 10-II-2015, G. Juárez leg., red entomológica, Bosque estacionalmente seco de llanura; 19 , Piura, Universidad de Piura, $05^{\circ} 10^{\prime} 11^{\prime \prime} \mathrm{S}-80^{\circ} 36^{\prime} 51^{\prime \prime} \mathrm{O}, 29 \mathrm{msnm}, 11$-III2015, U. González leg., red entomológica, Bosque estacionalmente seco de llanura; 1 , Sullana, Salitral, $04^{\circ} 51^{\prime} 27^{\prime \prime} \mathrm{S}-80^{\circ} 40^{\prime} 52^{\prime \prime} \mathrm{O}, 23 \mathrm{msnm}, 25-\mathrm{X}$ 2015, G. Juárez leg., red entomológica, Bosque estacionalmente seco de llanura; 1 q, Talara, Los Órganos, $04^{\circ} 10^{\prime} 38^{\prime \prime} \mathrm{S}-81^{\circ} 07^{\prime} 27^{\prime \prime} \mathrm{O}, 12 \mathrm{msnm}, 15-\mathrm{VI}-$ 2016, G. Juárez leg., red entomológica, Desierto; 1 ㅇ, Talara, Lobitos, $04^{\circ} 27^{\prime} 10^{\prime \prime} \mathrm{S}-81^{\circ} 16^{\prime} 40^{\prime \prime} \mathrm{O}, 12 \mathrm{msnm}$, 03-IV-2017, G. Juárez leg., red entomológica, desierto; 1 \& , Paita, La Huaca, 04 $54^{\prime} \mathrm{S}-80^{\circ} 57^{\prime} \mathrm{O}, 27 \mathrm{msnm}$, 17-XII-2015, U. González leg., red entomológica, Bosque estacionalmente seco de llanura; 1 \%, Sechura, Vice, 05²5'S-81 ${ }^{\circ} 46^{\prime} \mathrm{O}, 10$ msnm, 08-II-2018, U. González leg., red entomológica, Desierto; 1 đ̄, Sechura, Sechura, 05³4'S-81 ${ }^{\circ} 16^{\prime} \mathrm{O}, 16$ msnm, 11-IX-2018, U. González leg., red entomológica, Bosque estacionalmente seco de llanura; 1 ․ Morropón, Chulucanas, $05^{\circ} 05^{\prime} \mathrm{S}-80^{\circ} 99^{\prime} \mathrm{O}$, 350 msnm, 07-XI-2015, G. Juárez leg., red entomológica, Bosque estacionalmente seco de colina; 2 우, Morropón, Morropón, 0505'51"S-8009'44"O, 557 msnm, 04-IX2017, G. Juárez leg., red entomológica, Bosque estacionalmente seco de colina.

DistribuCión (Provincia) (paisaje ecológico). (PIU, SULL, TAL, PAI, SECH, MORR) (BES, DES).

Comentarios. Esta especie se distribuye en los valles costeros, secos y desérticos de Perú desde Tumbes en el norte hasta Lima en el sur (Rivera, 2001; Rivera \& Vergara-Cobián, 2017). Con los datos presentados aquí $P$. peruviana amplía su localización dentro de la región a las provincias de Piura, Talara, Paita y Sechura, anteriormente registrado para las provincias de Sullana y Morropón (Rivera \& Vergara-Cobián, 2017). Los individuos colectados fueron encontrados sobre hojas de vegetación arbórea de P.pallida y Colicodendron scabridum Kunth (Seem.), 1852 en bosques estacionalmente secos y desierto costero coincidiendo con el tipo de hábitat para esta especie.

REFERENCIA. Rivera \& Vergara-Cobián (2017).

ORDEN ORTHOPTERA Olivier, 1879

Suborden Ensifera Chopard, 1920

Familia Tettigoniidae Krauss, 1902

Subfamilia Conocephalinae Burmeister, 1838

Género Conocephalus Thunberg, 1815

10. Conocephalus sin identificar sp. 1 (Fig. 8)

Material eXAminado. 2 qq, Perú, Piura, La Arena, 05'20'34"S-8042'30"O, 29 msnm, 27-II-2015, red entomológica, G. Juárez, Bosque estacionalmente seco de llanura; 1 +, $1 \hat{\jmath}$, Piura, Universidad de Piura, $05^{\circ} 10^{\prime} 11^{\prime \prime} \mathrm{S}-80^{\circ} 36^{\prime} 51^{\prime \prime} \mathrm{O}, 29 \mathrm{msnm}, 07-\mathrm{IV}-2017$, trampa luz, U. González, Bosque estacionalmente seco de llanura; 1 ㅇ, Sullana, Salitral, 0451'27"S-80 40'52"O, 23 msnm, 11-II-2018, red entomológica, G. Juárez, Bosque estacionalmente seco de llanura; 1 , Sullana, Lancones, 043'ㄱ' $27^{\prime \prime} \mathrm{S}-80^{\circ} 32^{\prime} 55^{\prime \prime} \mathrm{O}, 190 \mathrm{msnm}, 12-\mathrm{V}-2015$, red entomológica, G. Juárez, Bosque estacionalmente seco de colina; 1 ㅇ, Talara, Los Órganos, $04^{\circ} 10^{\prime} 38^{\prime \prime} \mathrm{S}-81^{\circ} 07^{\prime} 27^{\prime \prime} \mathrm{O}$, 12 msnm, 09-IV-2018, red entomológica, G. Juárez, Desierto; 1 đ̊, Paita, $05^{\circ} 04^{\prime} \mathrm{S}-81^{\circ} 06^{\prime} \mathrm{O}, 10 \mathrm{msnm}, 23-\mathrm{XI}-$ 2018, red entomológica, G. Juárez, Bosque estacionalmente seco de llanura; $1 \delta^{\top}$, Sechura, Sechura, $05^{\circ} 34^{\prime} \mathrm{S}-81^{\circ} 16^{\prime} \mathrm{O}$, 16 msnm, 05-XII-2016, red entomológica, U. González, Bosque estacionalmente seco de llanura; 1 \&, Morropón, Chulucanas, $05^{\circ} 05^{\prime} \mathrm{S}-80^{\circ} 99^{\prime} \mathrm{O}, 350 \mathrm{msnm}, 22-\mathrm{I}-2015$, red entomológica, G. Juárez, Bosque estacionalmente seco de colina; 2 o , Morropón, Chalaco, $05^{\circ} 02^{\prime} 28^{\prime \prime} \mathrm{S}-79^{\circ} 47^{\prime} 45^{\prime \prime} \mathrm{O}$, 2025 msnm, 01-II-2015, red entomológica, G. Juárez, Bosque húmedo de montaña; $1 \delta$, Huancabamba, Huancabamba, $05^{\circ} 27^{\prime} 07^{\prime \prime} \mathrm{S}-79^{\circ} 36^{\prime} 22^{\prime \prime} \mathrm{O}, 2567 \mathrm{msnm}$, 27-X-2015, G. Juárez, Bosque húmedo de montaña;

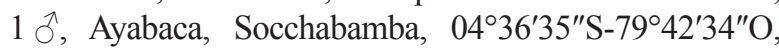
2755 msnm, 11-VII-2016, red entomológica, G. Juárez, Bosque húmedo de montaña; 1 , Ayabaca, Montero, 043'ㄴㄱ'S-7943'29"O, 1578 msnm, 19-VII-2016, red entomológica, U. González, Bosque seco interandino.

Distribución (Provincia) (paisaje ecológico). (PIU, SULL, TAL, PAI, SECH, MORR, HUA, AYA) (BES, DES, BSI, BHM).

Comentarios. Los individuos colectados fueron encontrados sobre hojas en vegetación arbórea, arbustiva y herbácea tanto áreas de bosques, algunos cultivos (arroz, maíz, caña de azúcar) y en zonas urbanizadas (jardines y parques).

Género Neoconocephalus Karny, 1907

11. Neoconocephalus sin identificar sp. 1 (Fig. 9)

Material examinado. 2 q, ,Perú, Piura, La Arena, $05^{\circ} 20^{\prime} 34^{\prime \prime} \mathrm{S}-80^{\circ} 42^{\prime} 30^{\prime \prime} \mathrm{O}, 29$ msnm, 27-II-2015, trampa luz, G. Juárez, Bosque estacionalmente seco de llanura; 
1 q, Sullana, Lancones, 04 $38^{\prime} 27^{\prime \prime} \mathrm{S}-80^{\circ} 32^{\prime} 55^{\prime \prime} \mathrm{O}$ 190 msnm, 12-V-2015, red entomológica, G. Juárez, Bosque estacionalmente seco de colina; 19 , Talara, Los Órganos, $04^{\circ} 10^{\prime} 38^{\prime \prime} \mathrm{S}-81^{\circ} 07^{\prime} 27^{\prime \prime} \mathrm{O}, 12 \mathrm{msnm}$, 09-IV-2018, red entomológica, G. Juárez, Desierto; $1 \delta^{\top}$, Paita, $05^{\circ} 04^{\prime} \mathrm{S}-81^{\circ} 06^{\prime} \mathrm{O}, 10 \mathrm{msnm}, 23-\mathrm{XI}-2018$, red entomológica, G. Juárez, Bosque estacionalmente seco de llanura; $1 \overbrace{}^{\lambda}$, Sechura, Sechura, $05^{\circ} 34^{\prime} \mathrm{S}-81^{\circ} 16^{\prime} \mathrm{O}, 16$ msnm, 05-XII-2016, red entomológica, U. González, Bosque estacionalmente seco de llanura; 1 , Morropón, Chulucanas, $05^{\circ} 05^{\prime} \mathrm{S}-80^{\circ} 99^{\prime} \mathrm{O}, 350 \mathrm{msnm}$, 22-I-2015, red entomológica, G. Juárez, Bosque estacionalmente seco de colina; 1 ते, Huancabamba, Sondor, 05 $18^{\prime} 56^{\prime \prime} \mathrm{S}-79^{\circ} 24^{\prime} 36^{\prime \prime} \mathrm{O}, 1755$ msnm, 12-VI-2016, G. Juárez, Bosque seco interandino; 1 đ, Ayabaca, Socchabamba, 0436'35"S-7942'34"O, 2755 msnm, 11-VII-2016, red entomológica, G. Juárez, Bosque húmedo de montaña.

DisTribuCión (Provincia) (paisaje ecológico). (PIU, SULL, TAL, PAI, SECH, MORR, HUA, AYA) (BES, DES, BSI, BHM).

Comentarios. Los individuos colectados fueron encontrados sobre hojas en vegetación arbórea y arbustiva tanto en áreas de bosques y zonas urbanizadas (jardines y parques).

Género Moncheca Walker, 1869

12. Moncheca $\sin$ identificar sp. 1

Material examinado. 1 , Perú, Huancabamba, El Carmen de la Frontera, $05^{\circ} 08^{\prime} 54^{\prime \prime} \mathrm{S}-79^{\circ} 45^{\prime} 21^{\prime \prime} \mathrm{O}, 2450 \mathrm{msnm}$, 02-X-2016, red entomológica, G. Juárez, Bosque húmedo de montaña.

Distribución (Provincia) (paisaje ecológico). (HUA) (BHM).

Comentarios. Las especies del género Moncheca se distribuyen en los bosques tropicales húmedos de México, Guatemala, Costa Rica, Guatemala, Panamá, Colombia, Ecuador, Perú y Bolivia (Montealegre-Zapata, 1997). El individuo colectado se encontró sobre vegetación arbustiva en bosque de neblina.

Subfamilia Phaneropterinae Burmeister, 1838

Género Ceraia Brunner von Wattenwyl, 1891

13. Ceraia $\sin$ identificar sp. 1

Material examinado. 1 , Perú, Piura, Piura, $05^{\circ} 12^{\prime} 03^{\prime \prime} \mathrm{S}-80^{\circ} 37^{\prime} 31^{\prime \prime} \mathrm{O}, 29 \mathrm{msnm}, 12-\mathrm{XI}-2016$, trampa luz, G. Juárez, Bosque estacionalmente seco de llanura; 1 o, Sullana, Salitral, $04^{\circ} 51^{\prime} 27^{\prime \prime} \mathrm{S}-80^{\circ} 40^{\prime} 52^{\prime \prime} \mathrm{O}$, 23 msnm, 12-XII-2015, red entomológica, G. Juárez, Bosque estacionalmente seco de llanura; 1 , Talara, Los Órganos, $04^{\circ} 10^{\prime} 38^{\prime \prime} \mathrm{S}-81^{\circ} 07^{\prime} 27^{\prime \prime} \mathrm{O}, 12 \mathrm{msnm}, 16-\mathrm{V}$ 2018, colecta manual, G. Juárez, Desierto; 1 J̄, Paita, Miguel Checa, $04^{\circ} 54^{\prime} \mathrm{S}-80^{\circ} 48^{\prime} \mathrm{O}, 17 \mathrm{msnm}, 17-\mathrm{VIII}-$ 2017, red entomológica, G. Juárez, Bosque estacionalmente seco de llanura; $1 \hat{\jmath}$, Sechura, Sechura, 05 $34^{\prime} \mathrm{S}-81^{\circ} 16^{\prime} \mathrm{O}, 16 \mathrm{msnm}, 05-\mathrm{II}-2015$, red entomológica, U. González, Bosque estacionalmente seco de llanura; 1 ㅇ, Morropón, Chulucanas, $05^{\circ} 05^{\prime} \mathrm{S}-80^{\circ} 99^{\prime} \mathrm{O}$, 350 msnm, 04-III-2017, colecta manual, G. Juárez, Bosque estacionalmente seco de colina.
Distribución (Provincia) (paisaje ecológico). (PIU, SULL, PAI, TAL, SECH, MORR) (BES, DES).

Comentarios. El género Ceraia tiene una amplia distribución en Centroamérica y Sudamérica (Grant, 1964). Los individuos colectados fueron encontrados sobre hojas en vegetación arbórea y arbustiva tanto en áreas de bosques y zonas urbanizadas.

Género Hetaira Brunner von Wattenwyl, 1891

14. Hetaira (Atopana) sin identificar sp. 1

Material eXAminado. 1 \% Perú, Huancabamba, Canchaque, 05²2'35"S - 79³6'23"O, 2000 msnm, 2-II-2018, red entomológica, U. González, Bosque húmedo de montaña.

Distribución (Provincia) (paisaje ecológico). (HUA) (BHM).

Género Scudderia Stål, 1873

15. Scudderia sin identificar sp. 1 (Fig. 10)

Material examinado. 1 \%, Perú, Piura, La Unión, 05²3'29"S-8044'58"O, 29 msnm, 04-IX-2015, red entomológica, G. Juárez, Bosque estacionalmente seco de llanura; 1 q, Sullana, Cabo Verde, 04 51'27"S-8040'52"O, 23 msnm, 07-X-2016, trampa luz, G. Juárez, Bosque estacionalmente seco de llanura; 1 q, Talara, Máncora, $04^{\circ} 06^{\prime} 26^{\prime \prime} \mathrm{S}-81^{\circ} 02^{\prime} 50^{\prime \prime} \mathrm{O}, 8$ msnm, 09-II-2017, colecta manual, G. Juárez, Desierto; $1 \partial^{\lambda}$, Paita, Miguel Checa, 045' $\mathrm{S}-80^{\circ} 48^{\prime} \mathrm{O}, 17 \mathrm{msnm}$, 11-VII-2018, red entomológica, G. Juárez, Bosque estacionalmente seco de llanura; $1 \hat{\jmath}$, Sechura, Sechura, $05^{\circ} 34^{\prime} \mathrm{S}-81^{\circ} 16^{\prime} \mathrm{O}, 16 \mathrm{msnm}, 15-\mathrm{VI}-2017$, red entomológica, U. González, Bosque estacionalmente seco de llanura; 1 + , Morropón, Chulucanas, $05^{\circ} 05^{\prime} \mathrm{S}-80^{\circ} 99^{\prime} \mathrm{O}$, 350 msnm, 14-VI-2016, colecta manual, G. Juárez, Bosque estacionalmente seco de colina.

DisTribuCión (Provincia) (paisaje ecológico). (PIU, SULL, PAI, TAL, SECH, MORR) (BES, DES).

Comentarios. Los individuos colectados fueron encontrados sobre hojas en vegetación arbórea y arbustiva en áreas de bosques y zonas urbanizadas.

Género Stilpnochlora Stål, 1873

16. Stilpnochlora $\sin$ identificar sp. 1 (Fig. 11)

Material eXAminado. 1 q, Perú, Huancabamba, Huancabamba, $05^{\circ} 24^{\prime} 07^{\prime \prime S}-79^{\circ} 36^{\prime 2} 2^{\prime \prime} \mathrm{O}, 2637 \mathrm{msnm}$, 22-VIII-2017, red entomológica, G. Juárez, Bosque húmedo de montaña.

DistRIBUCIÓN (Provincia) (paisaje ecológico). (HUA) (BHM).

Subfamilia Pterochrozinae Walker, 1871

Género Typophyllum Serville, 1838

17. Typophyllum sin identificar sp. 1 (Fig. 12)

Material eXaminado. 1 q, Perú, Ayabaca, Socchabamba,

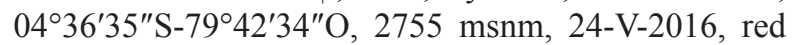
entomológica, G. Juárez, Bosque húmedo de montaña.

Distribución (Provincia) (paisaje ecológico). (AYA) (BHM).

Comentarios. Las especies del género Typophyllum se distribuyen desde el sur de México hasta el noroeste de Argentina habitando bosques húmedos tropicales y 
bosques andinos (Braun, 2015). El individuo colectado fue encontrado en bosque de neblina.

Familia Gryllidae Laicharting, 1781

Subfamilia Gryllinae Laicharting, 1781

Género Gryllus Linnaeus, 1758

18. Gryllus (Gryllus) capitatus Saussure, 1874

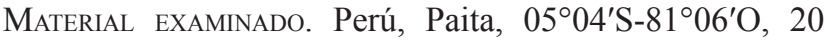
msnm, Bosque estacionalmente seco de llanura.

Distribución (Provincia) (paisaje ecológico). (PAI) (BES).

ReFERENCIA. Aguilar (1973).

Familia Gryllotalpidae Leach, 1815

Subfamilia Scapteriscinae Zuener, 1939

Género Neoscapteriscus Cadena-Castañeda, 2015

19. Neoscapteriscus in identificar sp. 1

Material eXAminado. 1 , Perú, Piura, La Arena, 0520'34"S-8042'30"O, 29 msnm, 12-X-2015, colecta manual, G. Juárez, Bosque estacionalmente seco de llanura; 1 ㅇ, Sullana, Lancones, $04^{\circ} 38^{\prime} 27^{\prime \prime} \mathrm{S}-80^{\circ} 32^{\prime} 55^{\prime \prime} \mathrm{O}$, 190 msnm, 09-IV-2015, colecta manual, G. Juárez, Bosque estacionalmente seco de colina; 1 \% , Talara, Los Órganos, $04^{\circ} 10^{\prime} 38^{\prime \prime} \mathrm{S}-81^{\circ} 07^{\prime} 27^{\prime \prime} \mathrm{O}, 12 \mathrm{msnm}, 19-\mathrm{VII}-$

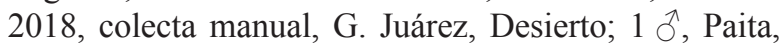
$05^{\circ} 04^{\prime} \mathrm{S}-81^{\circ} 06^{\prime} \mathrm{O}, 10 \mathrm{msnm}, 25-\mathrm{I}-2017$, colecta manual, G. Juárez, Bosque estacionalmente seco de llanura; 1 đै, Sechura, Sechura, $05^{\circ} 34^{\prime} \mathrm{S}-81^{\circ} 16^{\prime} \mathrm{O}, 16 \mathrm{msnm}, 13-\mathrm{XI}-$ 2017, colecta manual, U. González, Bosque estacionalmente seco de llanura; 1 \% , Morropón, Chulucanas, $05^{\circ} 05^{\prime} \mathrm{S}-80^{\circ} 99^{\prime} \mathrm{O}, 350 \mathrm{msnm}, 22-\mathrm{X}-2016$, colecta manual, G. Juárez, Bosque estacionalmente seco de colina; 1 శ̂,, Huancabamba, Sondor, 05¹8'56"S-79²4'36"O, 1755 msnm, 22-V-2015, G. Juárez, Bosque seco interandino; 1 ô, Ayabaca, Socchabamba, 04³6'35"S-7942'34"O, 2755 msnm, 19-VI-2016, colecta manual, G. Juárez, Bosque húmedo de montaña.

DisTRIBUCIÓN (Provincia) (paisaje ecológico). (PIU, SULL, TAL, PAI, SECH, MORR, HUA, AYA) (BES, DES, BSI, BHM).

Comentarios. Las especies del género Neoscapteriscus son netamente subterráneas alimentándose preferiblemente de raíces de plantas, tubérculos y tallos subterráneos causando en algunos casos serios daños a diversos cultivos. Los individuos colectados fueron encontrados en jardines con césped y en galerías subterráneas cerca a raíces de caña de azúcar, maíz y papa.

Familia Mogoplistidae Brunner von Wattenwyl, 1873

Subfamilia Mogoplistinae Brunner von Wattenwyl, 1873

Género Ornebius Guérin-Méneville, 1844

20. Ornebius peruviensis Chopard, 1913

Material eXaminado. Perú, Paita, $05^{\circ} 04^{\prime} \mathrm{S}-81^{\circ} 06^{\prime} \mathrm{O}$, $20 \mathrm{msnm}$, Bosque estacionalmente seco de llanura.

Distribución (Provincia) (paisaje ecológico). (PAI) (BES).

ReFERENCiA. Aguilar (1973).

Suborden Caelifera Ander, 1936

Familia Tridactylidae Brullé, 1835

Subfamilia Tridactylinae Brullé, 1835
Género Ellipes Scudder, 1902

21. Ellipes occidentalis Günther, 1977

Material examinado. 1 , Perú, Piura, Universidad de Piura, $05^{\circ} 10^{\prime} 11^{\prime \prime} \mathrm{S}-80^{\circ} 36^{\prime} 51^{\prime \prime} \mathrm{O}, 29 \mathrm{msnm}, \quad 04-\mathrm{IV}-$ 2015, U. González leg., trampa luz, Bosque estacionalmente seco de llanura; 19 , Sullana, Salitral, 04 51'27"S-80 40'52"O, 23 msnm, 28-X-2017, G. Juárez leg., colecta manual, Bosque estacionalmente seco de llanura; 19 , Sullana, Lancones, 043'ㄱ'ㄱ'S-80³2'55"O, 190 msnm, 04-VI-2015, G. Juárez leg., colecta manual, Bosque estacionalmente seco de colina; 1 q, Talara, El Alto, 04 ${ }^{\circ} 16^{\prime} 04^{\prime \prime} \mathrm{S}-81^{\circ} 13^{\prime} 09^{\prime \prime} \mathrm{O}$, 5 msnm, 23-IV-2015, G. Juárez leg., colecta manual, Desierto; 1 ㅇ, Paita, Miguel Checa, $04^{\circ} 54^{\prime} \mathrm{S}-80^{\circ} 48^{\prime} \mathrm{O}$, 10 msnm, 12-III-2016, G. Juárez leg., colecta manual, Bosque estacionalmente seco de llanura; 1 , , Sechura, Vice, $05^{\circ} 25^{\prime} \mathrm{S}-81^{\circ} 46^{\prime} \mathrm{O}, 10 \mathrm{msnm}, 17-\mathrm{VIII}-2015$, U. González leg., red entomológica, Desierto; 1 q, Morropón, Morropón, $05^{\circ} 05^{\prime} 51^{\prime \prime S}-80^{\circ} 09^{\prime} 44^{\prime \prime} \mathrm{O}, 557$ msnm, 14-V-2017, G. Juárez leg., trampa luz, Bosque estacionalmente seco de colina; $1 \hat{\jmath}$, Huancabamba, Huancabamba, $05^{\circ} 27^{\prime} 07^{\prime \prime} \mathrm{S}-79^{\circ} 36^{\prime} 22^{\prime \prime} \mathrm{O}, 2567$ msnm, 25-VII-2017, G. Juárez leg., colecta manual, Bosque húmedo de montaña; $2 \hat{\jmath} \hat{o}$, Ayabaca, Socchabamba, 0436'35"S-7942'34"O, 2755 msnm, 26-VII-2017, G. Juárez leg., red entomológica, Bosque húmedo de

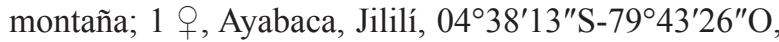
450 msnm, 09-X-2017, U. González leg., trampa luz, Bosque seco interandino.

DisTribuCión (Provincia) (paisaje ecológico). (PIU, SULL, TAL, PAI, SECH, MORR, AYA, HUA) (BES, DES, BSI, BHM).

Comentarios. La mayoría de los individuos colectados fueron encontrados principalmente sobre tierra húmeda, mientras que otros fueron capturados atraídos por la trampa luz.

Familia Proscopiidae Serville, 1838

Subfamilia Proscopiinae Serville, 1838

Género Anchotatus Brunner von Wattenwyl, 1890

22. Anchotatus brevicornis (Caudell, 1912) (Fig. 13)

Materialexaminado. 1 ,Perú,Piura, Castilla,Universidad Nacional de Piura, $05^{\circ} 10^{\prime} 51^{\prime \prime} \mathrm{S}-80^{\circ} 37^{\prime} 31^{\prime \prime} \mathrm{O}, 29 \mathrm{msnm}$, 17-IV-2018, U. González leg., red entomológica, Bosque estacionalmente seco de llanura; 1 , Piura, Universidad de Piura, $05^{\circ} 10^{\prime} 11^{\prime \prime} \mathrm{S}-80^{\circ} 36^{\prime} 51^{\prime \prime} \mathrm{O}, 29$ msnm, 16-VII-2018, G. Juárez leg., red entomológica, Bosque estacionalmente seco de llanura; 1 †, Sullana, Querecotillo, 0450'24"S-80³8'57"O, $23 \mathrm{msnm}$, 21-X-2017, G. Juárez leg., red entomológica, Bosque estacionalmente seco de llanura; 1 , Talara, Los Órganos, $04^{\circ} 10^{\prime} 38^{\prime \prime} \mathrm{S}-81^{\circ} 07^{\prime} 27^{\prime \prime} \mathrm{O}, 12$ msnm, 11-VIIi2017, G. Juárez leg., red entomológica, Bosque estacionalmente seco de llanura; 1 , Talara, El Alto, 04 $16^{\prime} 04^{\prime \prime} \mathrm{S}-81^{\circ} 13^{\prime} 09^{\prime \prime} \mathrm{O}, 8 \mathrm{msnm}, 03-\mathrm{IX}-2017, \mathrm{G}$. Juárez leg., red entomológica, Desierto; 1 q, Paita, $05^{\circ} 04^{\prime} \mathrm{S}-81^{\circ} 06^{\prime} \mathrm{O}, 10 \mathrm{msnm}, 02-\mathrm{VIII}-2017$, G. Juárez leg., red entomológica, Bosque estacionalmente seco

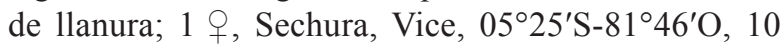


msnm, 20-II-2016, U. González leg., red entomológica, Desierto; 1 ô, Sechura, Sechura, $05^{\circ} 34^{\prime} \mathrm{S}-81^{\circ} 16^{\prime} \mathrm{O}, 16$ msnm, 13-XII-2017, U. González leg., red entomológica, Bosque estacionalmente seco de llanura; 1 , Morropón, Chulucanas, $05^{\circ} 05^{\prime} \mathrm{S}-80^{\circ} 99^{\prime} \mathrm{O}, 350 \mathrm{msnm}$, 14-VI-2017, G. Juárez leg., red entomológica, Bosque estacionalmente seco de colina; 2 우, Morropón, Morropón, $05^{\circ} 05^{\prime} 51^{\prime \prime} \mathrm{S}-80^{\circ} 09^{\prime} 44^{\prime \prime} \mathrm{O}, 557 \mathrm{msnm}$, 12-XI-2016, G. Juárez leg., red entomológica, Bosque estacionalmente seco de colina.

Distribución (Provincia) (paisaje ecológico). (PIU, SULL, TAL, PAI, SECH, MORR) (BES, DES).

Comentarios. Especie que se distribuye a lo largo de la franja costera de Perú específicamente en valles y lomas costeras, ecosistemas secos y desérticos (Aguilar, 1983). Los individuos colectados fueron encontrados sobre vegetación arbórea, arbustiva y herbácea en ecosistemas secos y desérticos, aunque también se colectaron sobre el suelo en áreas despejadas de bosques secos desprovistas de vegetación.

Familia Acrididae MacLeay, 1821

Subfamilia Ommatolampidinae Brunner von Wattenwyl, 1890

Género Abracris Walker, 1870

23. Abracris flavolineata (De Geer, 1773) (Fig. 14)

Material examinado. 1 9 , Perú, Piura, Huancabamba, Canchaque, $05^{\circ} 22^{\prime} 35^{\prime \prime} \mathrm{S}-79^{\circ} 36^{\prime} 26^{\prime \prime} \mathrm{O}, 2003 \mathrm{msnm}$, 13-VII-2015, G. Juárez leg., red entomológica, Bosque húmedo de montaña.

Distribución (Provincia) (paisaje ecológico). (HUA) (BHM).

Comentarios. El individuo colectado se encontró en vegetación herbácea a ras del suelo en bordes de bosque, lo que coincide con el tipo de hábitat mencionado para esta especie (Cadena-Castañeda \& Cardona-Granda, 2015).

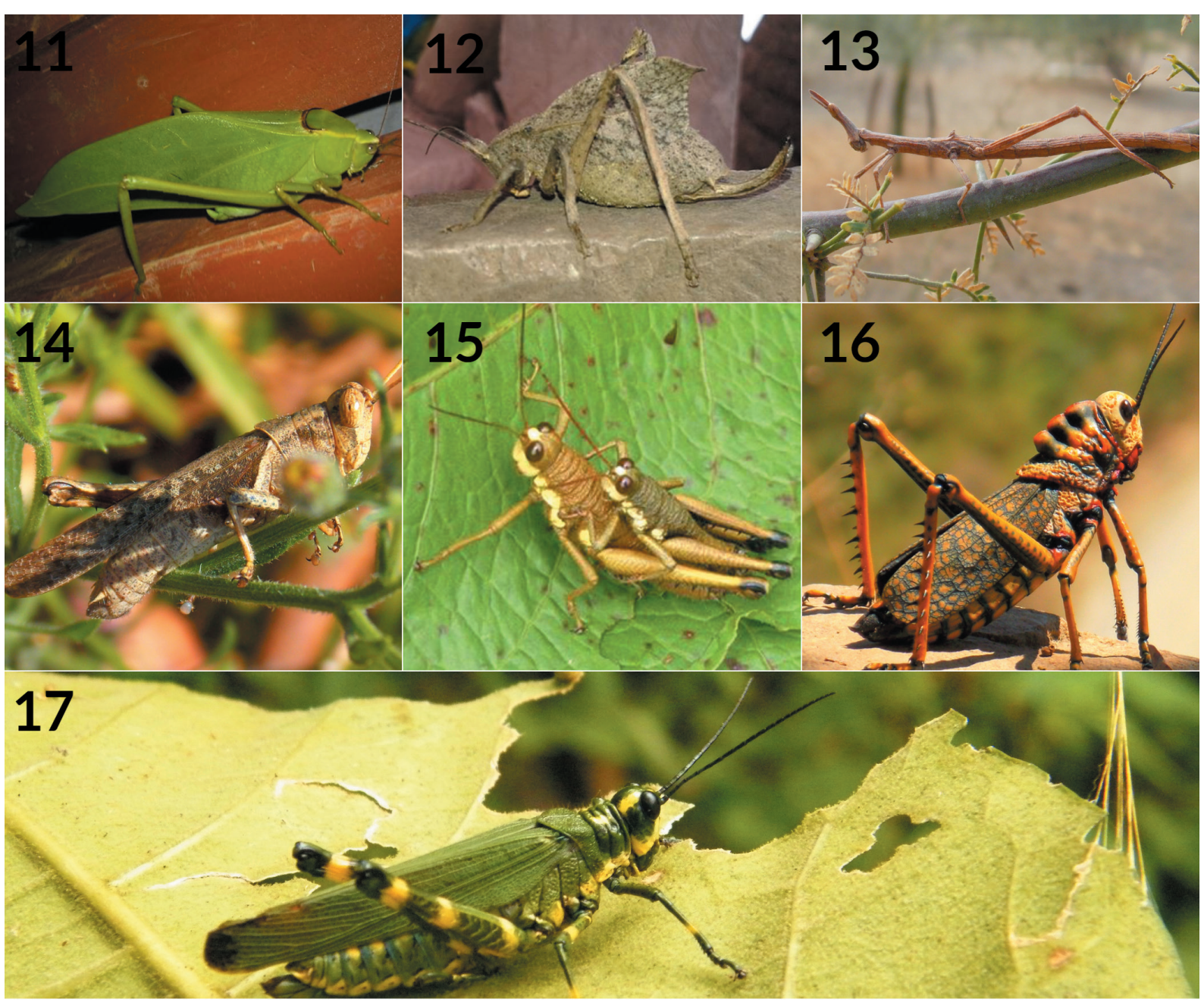

Figs. 11-17.- Habitus. 11. Stilpnochlora sin identificar sp. 1. 12. Typophyllum sin identificar sp. 1. 13. Anchotatus brevicornis (Caudell, 1912). 14. Abracris flavolineata (De Geer, 1773). 15. Nepiopteracris eburnifrons (Bruner, 1910). 16. Thrasyderes leprosus Bolivar, 1881. 17. Chromacris psittacus psittacus (Gerstaecker, 1873). [Fotografías 11, 13, 14, 16 de G. Juárez y U. González; Fotografías 12, 15, 17 de Danny Silva, J. Carrera, Peter Peña].

Figs. 11-17.- Habitus. 11. Stilpnochlora unidentified sp. 1. 12. Typophyllum unidentified sp. 1. 13. Anchotatus brevicornis (Caudell, 1912). 14. Abracris flavolineata (De Geer, 1773). 15. Nepiopteracris eburnifrons (Bruner, 1910). 16. Thrasyderes leprosus Bolívar, 1881. 17. Chromacris psittacus psittacus (Gerstaecker, 1873). [Photographs 11, 13, 14, 16 by G. Juárez and U. González; Photographs 12, 15, 17 by Danny Silva, J. Carrera, Peter Peña]. 
Género Nepiopteracris Carbonell \& Descamps, 1978 24. Nepiopteracris eburnifrons (Bruner, 1910) (Fig. 15)

Material eXaminado. 1 \%, Perú, Huancabamba, El Carmen de la frontera, $05^{\circ} 08^{\prime} 54^{\prime \prime} \mathrm{S}-79^{\circ} 25^{\prime} 41^{\prime \prime} \mathrm{O}, 2450 \mathrm{msnm}$, 16-VII-2015, G. Juárez leg., red entomológica, Bosque húmedo de montaña.

Distribución (Provincia) (paisaje ecológico). (HUA) (BHM).

Subfamilia Cyrtacanthacridinae Kirby, 1910

Género Schistocerca Stål, 1873

25. Schistocerca interrita Scudder, 1889

Material examinado. 3 o 9 , Perú, Piura, Catacaos, $05^{\circ} 15^{\prime} 42^{\prime \prime} \mathrm{S}-80^{\circ} 40^{\prime} 27^{\prime \prime} \mathrm{O}, 29 \mathrm{msnm}, 06-\mathrm{II}-2015$, U. González leg., red entomológica, Bosque estacionalmente seco de llanura; 3 우, $2 \overbrace{}^{\lambda}$, Piura, Universidad de Piura, 05 $10^{\prime} 11^{\prime \prime S}-80^{\circ} 36^{\prime} 51^{\prime \prime} \mathrm{O}, 29$ msnm, 26-II-2015, U. González leg., red entomológica, Bosque estacionalmente seco de llanura; 1 , , Piura, Castilla, Universidad Nacional de Piura, $05^{\circ} 10^{\prime} 51^{\prime \prime} \mathrm{S}-80^{\circ} 37^{\prime} 31^{\prime \prime} \mathrm{O}, \quad 29$ msnm, 14-III-2017, G. Juárez leg., red entomológica, Bosque estacionalmente seco de llanura; 1 , Sullana, Querecotillo, 0450'24"S-80³8'57"O, 23 msnm, 19-I-2016, G. Juárez leg., red entomológica, Bosque estacionalmente seco de llanura; 2 우, Sullana, Salitral, $04^{\circ} 51^{\prime} 27^{\prime \prime} \mathrm{S}-80^{\circ} 40^{\prime} 52^{\prime \prime} \mathrm{O}, 23 \mathrm{msnm}, 11-\mathrm{V}$ 2017, G. Juárez leg., red entomológica, Bosque estacionalmente seco de llanura; 1 , Sullana, Lancones,

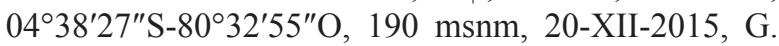
Juárez leg., red entomológica, Bosque estacionalmente seco de colina; 1 \% , Paita, $05^{\circ} 04^{\prime} \mathrm{S}-81^{\circ} 06^{\prime} \mathrm{O}, 10 \mathrm{msnm}$, 02-VIII-2017, G. Juárez leg., red entomológica, Bosque estacionalmente seco de llanura; 1 , Talara, Los Órganos, $04^{\circ} 10^{\prime} 38^{\prime \prime} \mathrm{S}-81^{\circ} 07^{\prime} 27^{\prime \prime} \mathrm{O}, 12 \mathrm{msnm}, 15-\mathrm{IV}-$ 2016, G. Juárez leg., red entomológica, Desierto; 1 q, Talara, El Alto, $04^{\circ} 16^{\prime} 04^{\prime \prime} \mathrm{S}-81^{\circ} 13^{\prime} 09^{\prime \prime} \mathrm{O}, 300$ msnm, 13-V-2016, red entomológica, G. Juárez leg., Bosque estacionalmente seco de colina; 2 + 9 , Paita, $05^{\circ} 04^{\prime} \mathrm{S}-81^{\circ} 06^{\prime} \mathrm{O}, 10 \mathrm{msnm}, 07-X I I-2016$, G. Juárez leg., red entomológica, Bosque estacionalmente seco de llanura; 1 q, Sechura, Vice, $05^{\circ} 25^{\prime} \mathrm{S}-81^{\circ} 46^{\prime} \mathrm{O}, 10$ msnm, 02-II-2015, U. González leg., red entomológica, Desierto; $1 \jmath^{\lambda}$, Sechura, Sechura, $05^{\circ} 34^{\prime} \mathrm{S}-81^{\circ} 16^{\prime} \mathrm{O}$, 16 msnm, 11-VII-2017, U. González leg., red entomológica, Bosque estacionalmente seco de llanura; 1 q, Morropón, Chulucanas, $05^{\circ} 05^{\prime} \mathrm{S}-80^{\circ} 99^{\prime} \mathrm{O}, 350$ msnm, 05-III-2017, G. Juárez leg., red entomoló-

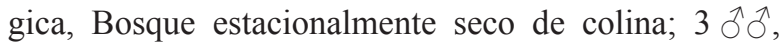
Huancabamba, Sondorillo, $05^{\circ} 24^{\prime} 07^{\prime \prime} \mathrm{S}-79^{\circ} 36^{\prime} 22^{\prime \prime} \mathrm{O}$, 1504 msnm, 22-IX-2016, G. Juárez leg., red entomológica, Bosque seco interandino; 1 त, Ayabaca, Suyo, 0430'45"S-8000'10"O, 408 msnm, 23-X-2015, G. Juárez leg., red entomológica, Bosque estacionalmente seco de colina.

DisTrIBUCIÓn (Provincia) (paisaje ecológico). (PIU, SULL, TAL, PAI, SECH, MORR, AYA, HUA) (BES, DES, BSI).

Comentarios. Especie de amplia distribución en la costa norte de Perú, sus poblaciones aumentan en la época lluviosa (diciembre-abril) convirtiéndose en algunos casos como plaga importante en cultivos (Beingolea, 1985; Pisfil, 1985). Los individuos colectados fueron encontrados en áreas de bosque entre vegetación arbórea, arbustiva y herbácea, sobre el suelo en áreas despejadas de bosques desprovistas de vegetación, en áreas urbanas y diversos cultivos (arroz, caña de azúcar, maíz).

Subfamilia Oedipodinae Walker, 1871

Género Trimerotropis Stål, 1873

26. Trimerotropis ochraceipennis reedi Liebermann, 1945

Material eXAminado. 1 , Perú, Piura, Castilla, Universidad Nacional de Piura, 05¹0'51"S-80³7'31"O, 29 msnm, 14-III-2017, G. Juárez leg., red entomológica, Bosque estacionalmente seco de llanura; 2 우, Salitral, 04 51'27"S-8040'52"O, 23 msnm, 11-V-2017, G. Juárez leg., red entomológica, Bosque estacionalmente seco de llanura; 1 q, Paita, $05^{\circ} 04^{\prime} \mathrm{S}-81^{\circ} 06^{\prime} \mathrm{O}, 10 \mathrm{msnm}$, 02-VIII-2017, G. Juárez leg., red entomológica, Bosque estacionalmente seco de llanura; 19 , Talara, Lobitos, 04 $27^{\prime} 10^{\prime \prime} \mathrm{S}-81^{\circ} 16^{\prime} 40^{\prime \prime} \mathrm{O}, 8$ msnm, 27-XI-2015, G. Juárez leg., red entomológica, desierto; 1 , Sechura, Vice, 05²5'S-81 ํ6' O, 10 msnm, 02-II-2015, U. González leg., red entomológica, Desierto; 1 đ̊, Sechura, Sechura, 05³4'S-81²16'O, 16 msnm, 11-VII-2017, U. González leg., red entomológica, Bosque estacionalmente seco de llanura; 1 q, Morropón, Chulucanas, $05^{\circ} 05^{\prime} \mathrm{S}-80^{\circ} 99^{\prime} \mathrm{O}$, 350 msnm, 05-III-2017, G. Juárez leg., red entomológica, Bosque estacionalmente seco de colina; 1 , Talara, El Alto, $04^{\circ} 16^{\prime} 04^{\prime \prime} \mathrm{S}-81^{\circ} 13^{\prime} 09^{\prime \prime} \mathrm{O}, 740 \mathrm{msnm}$, 12-V-2016, G. Juárez leg., red entomológica, bosque estacionalmente seco de colina.

Distribución (Provincia) (paisaje ecológico). (PIU, SULL, SECH, TAL) (BES, DES).

Comentarios. Especie que se distribuye en Chile y Perú (Rehn, 1939; Beingolea Guerrero, 1989). Los individuos colectados fueron encontrados en ambientes naturales especialmente en ecosistemas secos (desierto y bosques secos), urbanizados y cercanos a cultivos.

Subfamilia Melanoplinae Scudder, 1897

Género Jivarus Giglio-Tos, 1898

27. Jivarus pictifrons Ronderos, 1979

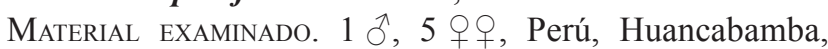
Canchaque, $05^{\circ} 22^{\prime} 35^{\prime \prime} \mathrm{S}-79^{\circ} 36^{\prime} 23^{\prime \prime} \mathrm{O}, 2300 \mathrm{msnm}$, 20-IX-2001, Amedegnato \& Poulain leg., bosque húmedo de montaña; $1 \hat{\jmath}$, Ayabaca, Bosque neblina de Cuyas, 04³6'S-7942'O, 2750 msnm, 24-VII-2016, red entomológica, G. Juárez leg., Bosque húmedo de montaña.

Distribución (Provincia) (paisaje ecológico). (HUA, AYA) (BHM).

Comentarios. Las especies del género Jivarus se restringen a los Andes de Perú, Ecuador y Colombia especialmente en bosques húmedos de montaña y paramos (Cigliano \& Amedegnato. 2010). J. pictifrons se distribuye en Ecuador y Perú de donde es citada solamente 
de la región Piura, específicamente de la provincia de Huancabamba (Cigliano \& Amedegnato. 2010). Con los datos presentados aquí, la especie amplía su localización dentro de la región hacia la provincia de Ayabaca. El individuo colectado se observó sobre vegetación herbácea densa en bosques de neblina, coincidiendo con el tipo de hábitat mencionado para esta especie (Cigliano \& Amedegnato. 2010).

28. Jivarus discoloris Cigliano \& Amedegnato, 2010

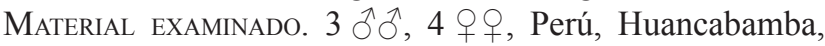
Carmen de la Frontera, $05^{\circ} 08^{\prime} 54^{\prime \prime} \mathrm{S}-79^{\circ} 25^{\prime} 41^{\prime \prime} \mathrm{O}, 3000$ msnm, 21-IX-2001, Amedegnato \& Poulain leg., bosque húmedo de montaña.

Distribución (Provincia) (paisaje ecológico). (HUA) (BHM).

Comentarios. Especie citada solamente de Perú y que se restringe como la especie anterior a los Andes, especialmente en bosques húmedos de montaña y paramos (Cigliano \& Amedegnato, 2010).

Referencia. Cigliano \& Amedegnato (2010).

Subfamilia Leptysminae Brunner von Wattenwyl, 1890

Género Stenacris Walker, 1870

29. Stenacris fissicauda chocoensis (Hebard 1923)

Material examinado. 1 jo, Perú, Sullana, 0454'S-8042'O, 28 msnm, 20-XII-2015, red entomológica, Bosque estacionalmente seco de llanura.

Distribución (Provincia) (paisaje ecológico). (SULL) (BES).

Comentarios. Especie que se distribuye desde Colombia hasta región costera del Pacífico en el extremo norte de Perú (Radclyffe, 1979).

REFERENCIA. Radclyffe (1979).

Subfamilia Gomphocerinae Fieber, 1853

Género Orphulella Giglio-Tos, 1894

30. Orphulella punctata (De Geer, 1773)

Material examinado. 1 q, Perú, Piura, Catacaos, 05 $15^{\prime} 42^{\prime \prime} \mathrm{S}-80^{\circ} 40^{\prime} 27^{\prime \prime} \mathrm{O}, 29 \mathrm{msnm}, 06-\mathrm{II}-2015, \mathrm{G}$. Juárez leg., red entomológica, Bosque estacionalmente seco de llanura; $290,1 \hat{0}$, Piura, Universidad de Piura, $05^{\circ} 10^{\prime} 11^{\prime \prime} \mathrm{S}-80^{\circ} 36^{\prime} 51^{\prime \prime} \mathrm{O}, 29 \mathrm{msnm}, 13-\mathrm{XI}-$ 2016, U. González leg., red entomológica, Bosque estacionalmente seco de llanura; 1 q, Piura, Castilla, Universidad Nacional de Piura, $05^{\circ} 10^{\prime} 51^{\prime \prime} \mathrm{S}-80^{\circ} 37^{\prime} 31^{\prime \prime} \mathrm{O}$, 29 msnm, 04-V-2017, G. Juárez leg., red entomológica, Bosque estacionalmente seco de llanura; 1 q, Sullana, Querecotillo, 04 50'24"S-80³8'57"O, 23 msnm, 29-IV2017, G. Juárez leg., red entomológica, Bosque estacionalmente seco de llanura; 2 ㅇ, Sullana, Salitral, 0451'27"S-8040'52"O, 23 msnm, 11-V-2017, G. Juárez leg., red entomológica, Bosque estacionalmente seco de llanura; 2 우, Paita, $05^{\circ} 04^{\prime} \mathrm{S}-81^{\circ} 06^{\prime} \mathrm{O}, 10 \mathrm{msnm}$, 17-III-2017, G. Juárez leg., red entomológica, Bosque estacionalmente seco de llanura; 1 , Sechura, Vice, 05²5'S-81²46'O, 10 msnm, 12-XI-2018, U. González leg., red entomológica, Desierto; $1 \AA$, Sechura, Sechura, $05^{\circ} 34^{\prime} \mathrm{S}-81^{\circ} 16^{\prime} \mathrm{O}$, 16 msnm, 15-IX-2016, U. González leg., red entomológica, Bosque estacionalmente seco de llanura; 1 ㅇ, Morropón, Chulucanas, $05^{\circ} 05^{\prime} \mathrm{S}-80^{\circ} 99^{\prime} \mathrm{O}$, 350 msnm, 13-X-2016, G. Juárez leg., , red entomológica, Bosque estacionalmente seco de colina; $1 \hat{\jmath}$, Ayabaca, Suyo, 04 $30^{\prime} 45^{\prime \prime} \mathrm{S}-80^{\circ} 00^{\prime} 10^{\prime \prime} \mathrm{O}, 408 \mathrm{msnm}$, 23-X-2015, G. Juárez leg., red entomológica, Bosque estacionalmente seco de colina.

DisTRIBUCIÓn (Provincia) (paisaje ecológico). (PIU, SULL, TAL, MORR, AYA) (BES, DES).

Comentarios. Esta especie se distribuye desde México hasta Argentina y es la más común de las especies del género Orphulella (Otte, 1979). Los individuos colectados se encontraron preferentemente entre la vegetación herbácea y sobre el suelo, incluso en áreas despejadas de bosques desprovistas de vegetación, coincidiendo con los tipos de hábitat mencionados para esta especie (Otte, 1979; Cassaro-Silva et al., 2011).

Familia Romaleidae Brunner von Wattenwyl, 1893

Subfamilia Romaleinae Pictec \& Saussure, 1887

Género Thrasyderes Bolívar, 1881

31. Thrasyderes leprosus Bolívar, 1881 (Fig. 16)

Material examinado. 1 q, Perú, Morropón, Piedra de El Toro, $05^{\circ} 11^{\prime} \mathrm{S}-79^{\circ} 55^{\prime} \mathrm{O}, 650$ msnm, 18-X-2015, G. Juárez leg., red entomológica, Bosque estacionalmente seco de

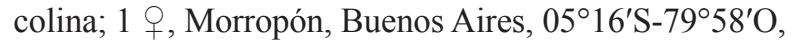
275 msnm, 20-I-2015, G. Juárez leg., red entomológica, Bosque estacionalmente seco de colina; 1 , , Sullana,

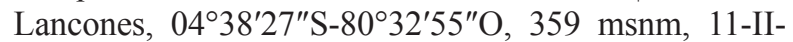
2015, G. Juárez leg., red entomológica, Bosque estacionalmente seco de colina; 1 , Sullana, Coto de Caza El Angolo, 04 $18^{\prime} 07^{\prime \prime} \mathrm{S}-80^{\circ} 47^{\prime} 47^{\prime \prime} \mathrm{O}, 400 \mathrm{msnm}, 11-\mathrm{II}-$ 2015, G. Juárez leg., red entomológica, Bosque estacionalmente seco de colina; $1 \hat{\jmath}$, Huancabamba, Canchaque, 05'22'35"S-79³6'23"O, 1000 msnm, 03-VIII-2016, U. González leg., red entomológica, Bosque seco interan-

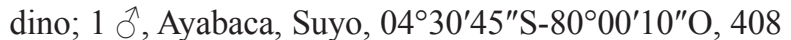
msnm, 13-VII-2016, G. Juárez leg., red entomológica, Bosque estacionalmente seco de colina; $1 \hat{\partial}$, Ayabaca, Simbaca, 04²7'98'S-7954'22"O, 1991 msnm, 13-VII2017, G. Juárez leg., red entomológica, Bosque húmedo de montaña.

Distribución (Provincia) (paisaje ecológico). (SULL, MORR, HUA, AYA) (BES, BSI).

Comentarios. Especie que se distribuye en Ecuador en la provincia de Guayas (Rehn, 1944; Buzzetti \& Carotti, 2008) y Perú (Beingolea-Guerrero, 1989). Para Perú esta especie es citada por Beingolea-Guerrero (1989), pero no cuenta con registros de localidades de colecta siendo esta la única cita existente en la literatura para el territorio peruano. En consecuencia presentamos los primeros registros de localidades exactas para esta especie en Perú, los mismos que se distribuyen hasta el momento en bosques estacionalmente secos, bosques secos interandinos, bosques húmedos de montaña entre los $270 \mathrm{~m}$ a $2000 \mathrm{~m}$ de altitud. Los individuos colectados fueron encontrados sobre la vegetación arbórea (preferentemente sobre ramas y troncos), herbácea y sobre el suelo. 
Género Chromacris Walker, 1870

32. Chromacris psittacus psittacus (Gerstaecker, 1873) (Fig. 17)

Material examinado. 1 , Perú, Morropón, Piedra de El Toro, $05^{\circ} 11^{\prime} \mathrm{S}-79^{\circ} 55^{\prime} \mathrm{O}, 650$ msnm, 25-V-2016, G. Juárez leg., red entomológica, Bosque estacionalmente seco de colina; 1 + , Morropón, Caracucho, $05^{\circ} 11^{\prime} \mathrm{S}-79^{\circ} 55^{\prime} \mathrm{O}$, 750 msnm, 13-III-2015, G. Juárez leg., red entomológica, Bosque estacionalmente seco de colina; 1 , Sullana, Lancones, $04^{\circ} 08^{\prime} 52^{\prime \prime} \mathrm{S}-80^{\circ} 35^{\prime} 24^{\prime \prime} \mathrm{O}, 359 \mathrm{msnm}$, 11-II-2015, G. Juárez leg., red entomológica, Bosque estacionalmente seco de colina; 1 , Sullana, Coto de Caza El Angolo, 04 $18^{\prime} 07^{\prime \prime S}-80^{\circ} 47^{\prime} 47^{\prime \prime} \mathrm{O}, 400 \mathrm{msnm}$, 11-II-2015, G. Juárez leg., red entomológica, Bosque estacionalmente seco de colina.

Distribución (Provincia) (paisaje ecológico). (MORR, SULL) (BES).

Comentarios. NuevoregistroparaPerú. Elgénero Chromacris es netamente neotropical y está compuesto por 11 especies distribuidas desde México hasta Argentina. En Perú se registran tres especies: Chromacris peruviana (Pictec \& Saussure, 1887), Chromacris icterus (Pictec \& Saussure, 1887) y Chromacris speciosa (Thunberg, 1824) (Radclyffe \& Carbonell, 1982). Chromacris psittacus Gerstaecker, 1873 contiene dos subespecies: C. psittacus psittacus distribuida en Costa Rica, Panamá, Venezuela, Colombia y Ecuador (Radclyffe \& Carbonell, 1982; Cigliano et al., 2018) y Chromacris psittacus pacificus Roberts \& Carbonell, 1982 distribuida en Colombia y Ecuador (Radclyffe \& Carbonell, 1982; Cadena-Castañeda et al., 2016; Cigliano et al., 2018). Las dos subespecies se pueden diferencian básicamente por la banda amarilla proximal del fémur posterior que en $C$. psittacus psittacus no es entera estando interrumpida por la banda verde adyacente, mientras que en C. psittacus pacificus la banda amarilla está completamente entera (Radclyffe \& Carbonell, 1982). Hasta el momento C. psittacus psittacus en Perú se distribuye en bosques estacionalmente secos a partir $350 \mathrm{~m}$ hasta los $800 \mathrm{~m}$ de altitud, siendo más abundante en épocas lluviosas (diciembre-abril), lo que coincide con los tipos de hábitat citados para las especies del género Chromacris (Radclyffe \& Carbonell, 1982). Los individuos colectados fueron encontrados sobre vegetación arbórea y arbustiva preferentemente sobre hojas.

\section{Agradecimientos}

A Julio Rivera (Mantidae), Rudolf Scheffrahn (Kalotermitidae) y Oscar Cadena Castañeda (Orthoptera) por el envío de material bibliográfico y ayuda en la identificación de algunos especies.

\section{Referencias}

Aguilar, P. G., 1973. Especies de grillos registrados para Perú. Revista Peruana de Entomología, 16(1): 121-123.

Aguilar, P. G., 1983. Especies de proscópidos registrados para el Perú. Revista Peruana de Entomología, 26(1): $85-88$.
Beccaloni, G. \& Eggleton, P., 2013. Order Blattodea. In: Zhang, Z.-Q. (ed.). Animal Biodiversity: An Outline of Higher-level Classification and Survey of Taxonomic Richness (Addenda 2013). Zootaxa, 3703(1): 46-48. https://doi.org/10.11646/zootaxa.3703.1.10

Beccaloni, G. W., 2014. Cockroach Species File Online. Version 5.0/5.0. Disponible en http://Cockroach. SpeciesFile.org (consultada 14 Nov. 2019).

Beingolea, O. D., 1985. La langosta Schistocerca interrita en la costa norte de Perú, durante 1983. Revista Peruana de Entomología, 28: 35-40.

Beingolea-Guerrero, O., 1989. Lista de acrídidos (OrthopteraCaelifera: Acrididae), registrados para el Perú. Revista Peruana de Entomología, 32: 37-40.

Bouchard, P., Bousquet, Y., Davies, A., Alonso-Zarazaga, M. A., Lawrence, J., Lyal, C., Newton, A., Reid, C., Schmitt, M., Ślipiński, A. \& Smith, A., 2011. Familygroup names in Coleoptera (Insecta). ZooKeys, 88: 1-972. https://doi.org/10.3897/zookeys.88.807

Braun, H., 2015. Little walking leaves from southeast Ecuador: biology and taxonomy of Typophyllum species (Orthoptera, Tettigoniidae, Pterochrozinae). Zootaxa, 4012(1): 1-32. https://doi.org/10.11646/zootaxa.4012.1.1

Buzzetti, F \& Carotti, G., 2008. Annotated list of the Caelifera of Ecuador (Insecta: Orthoptera). In: World Biodiversity Association (eds.). Biodiversity of South America Vol. 1. Italy: 39-66.

Cassaro-Silva, M., Serrão, J. E., Sousa-Silva, C. \& Marques-Pacheco, J., 2001. Identificacão de predadores de Orphulella punctata (de Geer) (Orthoptera, Acrididae) através da serologia. Revista Brasileira de Zoologia, 18(1): 75-79.

Cadena-Castañeda, O. J., 2015. The phylogeny of mole crickets (Orthoptera: Gryllotalpoidea: Gryllotalpidae). Zootaxa, 3985(4): 451-490. https://doi.org/10.11646/ zootaxa.3985.4.1

Cadena-Castañeda, O. J. \& Cardona-Granda, J.M., 2015. Introducción a los saltamontes de Colombia (Orthoptera: Caelifera, Acridomorpha, Tetrigoidea \& Tridactyloidea). Lulu.com. Colombia. 536 pp.

Cadena-Castañeda, O. J., Gutiérrez, Y- \& Bacca, T., 2016. New and little known Orthoptera (Ensifera and Caelifera) from the Ñambí River Natural Reserve, Nariño, Colombia. Zootaxa, 4162(2): 201-224. https:// doi.org/10.11646/zootaxa.4162.2.1

Carbonell, C. S. \& Descamps, M., 1978. Revue des Ommatolampae (Acridoidea, Ommatolampinae). Annales de la Société Entomologique de France, 14(1): 1-35.

Chaboo, C., 2015. Beetles (Coleoptera) of Peru: A Survey of the Families. Part I. Overview. Journal of the Kansas Entomological Society, 88(2): 135-139. https://doi. org/10.2317/0022-8567-88.2.135

Caudell, A. N., 1912. A new Proscopiid Grasshopper from Peru. Psyche, A Journal of Entomology, 19: 12-13. https://doi.org/10.1155/1912/56456

Cigliano, M. M. \& Amedegnato, C., 2010. The high-Andean Jivarus Giglio-Tos (Orthoptera, Acridoidea, Melanoplinae): systematics, phylogenetic and biogeographic considerations. Systematic Entomology, 35: 692721. https://doi.org/10.1111/j.1365-3113.2010.00538.x 
Cigliano, M. M., Braun, H., Eades, E. C. \& Otte, D., 2018. Orthoptera Species File Online. Version 5.0/5.0. Disponible en http://orthoptera.speciesfile.org (consultada 21 Nov. 2019).

Fernández, M., Martínez, D., Tantaleán, M. \& Martínez, R., 2001. Parasitos presentes en Periplaneta americana Linnaeus "cucaracha domestica" de la ciudad de Ica. Revista Peruana de Biología, 8(2): 105-113.

Fernández, S. \& Pujade-Villar, J., 2015. Orden Hymenoptera. RevistaIde@-SEA, 59: 1-36.

Grant, H. J., 1964. Revision of the Genera Ceraia and Euceraia, with Notes on Their Relationship to Scudderia (Orthoptera; Tettigoniidae; Phaneropterinae). Proceedings of the Academy of Natural Sciences of Philadelphia, 116: 29-117. https://www.jstor.org/ stable/4064622

Iannacone, J. \& Alvariño, L., 2007. Integración del control químico y etológico para la supresión poblacional de Blattellagermanica (Linnaeus) (Dictyoptera: Blattellidae) en Lima, Perú. Parasitología Latinoamericana, 62: 7-15.

Ingrisch, S. 2011. Order Orthoptera Oliver, 1789. In: Zhang, Z.-Q. (ed.). Animal biodiversity: An Outline of higher-level classification and survey of taxonomic richness. Zootaxa, 3148: 195-197. https://doi.org/10.11646/ zootaxa.3148.1.35

Juárez-Noé, G. \& González-Coronado, U., 2018. Listado de himenópteros (Insecta: Hymenoptera) de la región Piura, Perú. Folia Entomológica Mexicana (n.s.), 4(2): 48-65.

Juárez-Noé, G. \& González-Coronado, U., 2019. Actualización a la lista de coleópteros (Insecta: Coleoptera) de la región Piura, Perú. Graellsia, 75(2): e097 [1-37]. https://doi.org/10.3989/graellsia.2019. v75.233

Klass, K. \& Meier, R., 2006. A phylogenetic analysis of Dictyoptera (Insecta) based on morphological characters. Entomologische Abhandlungen, 63(1-2): 3-50.

Krishna, K., Grimaldi, D., Krishna, V. \& Engel, M. 2013. Treatise on the Isoptera of the world. Introduction. Bulletin of the American Museum of Natural History, 377: 1-200.

Márquez-Luna, J., 2005. Técnicas de colecta y preservación de insectos. Boletín de la Sociedad Entomológica Aragonesa (S.E.A.), 37: 385-408.

MINAM (Ministerio del Ambiente), 2018. Mapa Nacional de Ecosistemas Del Perú. Memoria Descriptiva. Ministerio del Ambiente. Lima. 60 pp.

Mizukubo, T., 1981. A revision of the genus Blattella (Blattaria: Blattellidae) of Japan, I. Terminology of the male genitalia and description of a new species from Okinawa Island. Esakia, 17: 149-159.

Montealegre-Zapata, F., 1997. Estudio de la fauna de Tettigoniidae (Orthoptera: Ensifera). Tesis para título de Biólogo entomólogo. Universidad del Valle, CaliColombia. 292 pp.

More, A. Villegas, P. \& Alzamora, M., 2014. Piura, Áreas prioritarias para la conservación de la biodiversidad. Naturaleza y Cultura Internacional-PROFONANPE, Lima, Perú, 166 pp.

Otte, D., 1979. Revision of the Grasshopper Tribe Orphulellini (Gomphocerinae: Acrididae). Proceedings of the Academy of Natural Sciences of Philadelphia, 131: 52-88. https://www.jstor.org/stable/4064782

Otte, D., Spearman, L. \& Stiewe, M. B. D., 2018. Mantodea Species File Online. Version 5.0/5.0 (disponible en http:/Mantodea.speciesfile.org)

Pellens, R. \& Grandcolas, P., 2002. Are successful colonizers necessarily invasive species? The case of the so-called invading parthenogenetic cockroach, Pycnoscelus surinamensis, in the Brazilian Atlantic forest. Revue d'Ecologie (Terre Vie), 57: 253-261.

Pisfil-Llontop, A., 1985. La langosta Schistocerca interrita como plaga en Lambayeque durante 1983-1984. Revista Peruana de Entomología, 28: 41-45.

Radclyffe, H., 1979. A Revision of the Tribe Leptysmini except the Genus Cylindrotettix (Orthoptera: Acrididae: Leptysminae). Proceedings of the Academy of Natural Sciences of Philadelphia, 129: 33-69. https://www. jstor.org/stable/4064738.

Radclyffe, H. \& Carbonell, C. S., 1982. A revision of the grasshopper genera Chromacris and Xestotrachelus (Orthoptera, Romaleidae, Romaleinae). Proceedings of the California Academy of Sciences, 43(4): 43-58.

Ramírez-Pérez, J., 1989. La cucaracha como vector de agentes patógenos. Boletín de la Oficina Sanitaria Latinoamericana, 107(1): 41-53.

Rehn, J. A., 1939. The South American Species of the Oedipodine Genus Trimerotropis (Orthoptera: Acrididae). Transactions of the American Entomological Society, 65: 395-414. http://www.jstor.org/stable/25077447

Rehn, J. A. G., 1944. The genera Aplatacris and Thrasyderes (Orthoptera; Acrididae; Romaleinae; Tropidacres). Notulae Naturae, 131: 1-12.

Rivera, J., 2001. Sobre la identidad de Vates peruviana Rehn, 1911 (Mantodea: Vatidae). Revista Peruana de Entomología, 42: 93-96.

Rivera, J., 2010. A historical review of praying mantid taxonomy and systematics in the Neotropical Region: State of knowledge and recent advances (Insecta: Mantodea). Zootaxa, 2638: 44-64. http://dx.doi.org/10.11646/zootaxa.2638.1.3

Rivera, J., Yagui, H. \& Ehrmann, R., 2011. Mantids in the MistTaxonomy of the Andean genus Pseudopogonogaster Beier, 1942, a cloud forest specialist, with notes on its biogeography and ecology (Mantodea: Thespidae: Miopteryginae). Insect Systematics \& Evolution, 42: 313-335. https://doi.org/10.1163/187631211X595056

Rivera, J. \& Vergara-Cobián, C., 2017. A checklist of the praying mantises of Peru: new records, one new genus (Piscomantis gen. n.) and biogeographic remarks (Insecta, Mantodea). Zootaxa, 4337(3): 361-389. https://doi.org/10.11646/zootaxa.4337.3.3

Roth, L., 1998. The Cockroach genus Pycnoscelus Scudder, with a description of Pycnoscelus femapterus, sp. nov. (Blattaria: Blaberidae: Pycnoscelinae). Oriental Insects, 32: 93-130. https://doi.org/10.1080/00305316.1998.10 433770

Schapheer, C., Sandoval, G. \& Villagra, C. A., 2018. Pest cockroaches may Overcome environmental restriction due to anthropization. Journal of Medical Entomology, 55(5): 1357-1364. https://doi.org/10.1093/jme/tjy090 
Scheffrahn, R., 1994. Incisitermes furvus, a New Drywood Termite (Isoptera: Kalotermitidae) from Puerto Rico. The Florida Entomologist, 77(3): 365-372. https://doi. org/10.2307/3496106

Song, H., 2009. Schistocerca Information Site. Taxonomic Identification Key to Schistocerca species (disponible en https://www.schistocerca.org/key.htm).

Svenson, G., 2014. Revision of the Neotropical bark mantis genus Liturgusa Saussure, 1869 (Insecta, Mantodea,
Liturgusini). ZooKeys, 390: 1-214. https://doi. org/10.3897/zookeys.390.6661

Taucare-Ríos, A., Carvajal, M. \& Faúndez, E. I., 2018. Primer registro de Pycnoscelus surinamensis (Linnaeus, 1758) (Blattodea: Blaberidae) en Chile Continental. Boletín de la Sociedad Entomológica Aragonesa (S.E.A.), 63: 240-242.

Torres, F., 2015. Orden Blattodea. Revista Ide@-SEA, 48: $1-13$. 
Apéndice 1.- Localidades de muestreo de colectas de Mantodea, Blattodea y Orthoptera en la región Piura, Perú.

Appendix 1.- Sampling localities of the Mantodea, Blattodea and Orthoptera collections in the Piura region, Peru.

\begin{tabular}{|c|c|c|c|c|}
\hline Provincia & Localidad de muestreo & Coordenadas & Paisaje ecológico & Altura (m.s.n.m) \\
\hline \multirow[t]{7}{*}{ Piura } & Ciudad de Piura & $05^{\circ} 12^{\prime} 03^{\prime \prime} \mathrm{S}-80^{\circ} 37^{\prime} 31^{\prime \prime O}$ & BESII & 30 \\
\hline & Universidad Nacional de Piura & $05^{\circ} 10^{\prime} 51^{\prime \prime} \mathrm{S}-80^{\circ} 37^{\prime} 08^{\prime \prime} \mathrm{O}$ & BESII & 30 \\
\hline & Universidad de Piura & $05^{\circ} 10^{\prime} 11^{\prime \prime S}-80^{\circ} 36^{\prime} 51^{\prime \prime O}$ & BESII & 30 \\
\hline & Distrito Catacaos & $05^{\circ} 15^{\prime} 55^{\prime \prime} \mathrm{S}-80^{\circ} 40^{\prime} 30^{\prime \prime} \mathrm{O}$ & BESII & 30 \\
\hline & Distrito La Unión & $05^{\circ} 23^{\prime} 29^{\prime \prime} \mathrm{S}-80^{\circ} 44^{\prime} 58^{\prime \prime} \mathrm{O}$ & BESII & 30 \\
\hline & Distrito La Arena & $05^{\circ} 20^{\prime} 34^{\prime \prime} \mathrm{S}-80^{\circ} 42^{\prime} 30^{\prime \prime} \mathrm{O}$ & BESII & 30 \\
\hline & Distrito Castilla & $05^{\circ} 11^{\prime} 05^{\prime \prime S}-80^{\circ} 57^{\prime} 27^{\prime \prime O}$ & BESII & 30 \\
\hline \multirow[t]{7}{*}{ Sullana } & Distrito Cabo Verde & $04^{\circ} 51^{\prime} 27^{\prime \prime} \mathrm{S}-80^{\circ} 40^{\prime} 52^{\prime \prime O}$ & BESII & 40 \\
\hline & Distrito Salitral & $04^{\circ} 51^{\prime} 27^{\prime \prime} \mathrm{S}-80^{\circ} 40^{\prime} 52^{\prime \prime} \mathrm{O}$ & BESII & 40 \\
\hline & Caserío Cabo Verde & $04^{\circ} 51^{\prime} 27^{\prime \prime} \mathrm{S}-80^{\circ} 40^{\prime} 52^{\prime \prime} \mathrm{O}$ & BESII & 40 \\
\hline & Distrito Lancones & $04^{\circ} 38^{\prime} 27^{\prime \prime} \mathrm{S}-80^{\circ} 32^{\prime} 55^{\prime \prime O}$ & BESc/BESm & $190-1500$ \\
\hline & Distrito Querecotillo & $04^{\circ} 50^{\prime} 24^{\prime \prime} \mathrm{S}-80^{\circ} 38^{\prime} 57^{\prime \prime O}$ & BESII & 40 \\
\hline & Coto de Caza El Angolo & $04^{\circ} 18^{\prime} 07^{\prime \prime} \mathrm{S}-80^{\circ} 47^{\prime} 47^{\prime \prime O}$ & BESc/BESm & $500-1500$ \\
\hline & Distrito Miguel Checa & $04^{\circ} 54^{\prime} 08^{\prime \prime} \mathrm{S}-80^{\circ} 48^{\prime} 56^{\prime \prime O}$ & BESII/DES & $0-20$ \\
\hline \multirow[t]{3}{*}{ Paita } & Ciudad de Paita & $05^{\circ} 04^{\prime} \mathrm{S}-81^{\circ} 06^{\prime} \mathrm{O}$ & BESII/DES & $0-20$ \\
\hline & Distrito La Huaca & $04^{\circ} 54^{\prime} 36^{\prime \prime} \mathrm{S}-80^{\circ} 57^{\prime} 42^{\prime \prime O}$ & BESII & 20 \\
\hline & Distrito Vichayal & $04^{\circ} 51^{\prime} 55^{\prime \prime} \mathrm{S}-81^{\circ} 04^{\prime} 19^{\prime \prime O}$ & BESII/DES & $0-20$ \\
\hline \multirow[t]{4}{*}{ Talara } & Distrito El Alto & $04^{\circ} 16^{\prime} 04^{\prime \prime} \mathrm{S}-81^{\circ} 13^{\prime} 09^{\prime \prime} \mathrm{O}$ & BESc/BESm & $700-1200$ \\
\hline & Distrito Los Órganos & $04^{\circ} 10^{\prime} 38^{\prime \prime} \mathrm{S}-81^{\circ} 07^{\prime} 29^{\prime \prime} \mathrm{O}$ & $\mathrm{BESc} / \mathrm{BESm}$ & $600-1450$ \\
\hline & Distrito Lobitos & $04^{\circ} 27^{\prime} 10^{\prime \prime} \mathrm{S}-81^{\circ} 16^{\prime} 40^{\prime \prime O}$ & BESII/DES & $0-200$ \\
\hline & Distrito Máncora & $04^{\circ} 06^{\prime} 26^{\prime \prime} \mathrm{S}-81^{\circ} 02^{\prime} 50^{\prime \prime O}$ & $\mathrm{BESc/BESm}$ & $750 / 1500$ \\
\hline \multirow[t]{3}{*}{ Sechura } & Ciudad de Sechura & $05^{\circ} 34^{\prime} \mathrm{S}-80^{\circ} 49^{\prime} \mathrm{O}$ & BESII/DES & $0-17$ \\
\hline & Desierto de Sechura & $05^{\circ} 49^{\prime} 60^{\prime \prime} \mathrm{S}-80^{\circ} 40^{\prime} \mathrm{O}$ & DES & $0-20$ \\
\hline & Distrito Vice & $05^{\circ} 25^{\prime} 18^{\prime \prime} \mathrm{S}-80^{\circ} 46^{\prime} \mathrm{O}$ & BESII/DES & $0-10$ \\
\hline \multirow[t]{8}{*}{ Morropón } & Ciudad Morropón & $05^{\circ} 05^{\prime} 51^{\prime \prime} \mathrm{S}-80^{\circ} 09^{\prime} 44^{\prime \prime O}$ & BESc/BESm & $500-1100$ \\
\hline & Caserío Piedra del Toro & $05^{\circ} 11^{\prime} \mathrm{S}-79^{\circ} 55^{\prime} \mathrm{O}$ & BESc/BESm & $550-1400$ \\
\hline & Distrito Chulucanas & $05^{\circ} 05^{\prime} 33^{\prime \prime} \mathrm{S}-80^{\circ} 09^{\prime} 45^{\prime \prime} \mathrm{O}$ & BESII/BESc & $200-1000$ \\
\hline & Distrito Santo Domingo & $05^{\circ} 01^{\prime} 45^{\prime \prime} \mathrm{S}-79^{\circ} 52^{\prime} 34^{\prime \prime O}$ & $\mathrm{BHM}$ & $2200-3100$ \\
\hline & Distrito Chalaco & $05^{\circ} 02^{\prime} 28^{\prime \prime S}-79^{\circ} 47^{\prime} 45^{\prime \prime O}$ & $\mathrm{BHM}$ & $2000-3000$ \\
\hline & Distrito Montero & $05^{\circ} 10^{\prime} 51^{\prime \prime} \mathrm{S}-79^{\circ} 45^{\prime} 02^{\prime \prime O}$ & $\mathrm{BHM}$ & $2000-2200$ \\
\hline & Distrito Buenos Aires & $05^{\circ} 66^{\prime} 02^{\prime \prime} \mathrm{S}-79^{\circ} 58^{\prime} 03^{\prime \prime} \mathrm{O}$ & $\mathrm{BESc} / \mathrm{BESm}$ & $500-1100$ \\
\hline & Caserío Caracucho & $05^{\circ} 14^{\prime} 02^{\prime \prime S}-79^{\circ} 91^{\prime} 56^{\prime \prime O}$ & BESc & $500-850$ \\
\hline \multirow[t]{5}{*}{ Huancabamba } & Distrito Canchaque & $05^{\circ} 22^{\prime} 35^{\prime \prime} \mathrm{S}-79^{\circ} 36^{\prime} 23^{\prime \prime} \mathrm{O}$ & $\mathrm{BHM}$ & $2000-2800$ \\
\hline & Distrito Huancabamba & $05^{\circ} 24^{\prime} 07^{\prime \prime} \mathrm{S}-79^{\circ} 36^{\prime} 22^{\prime \prime O}$ & $\mathrm{BHM}$ & $2500-3100$ \\
\hline & Distrito Sondorillo & $05^{\circ} 24^{\prime} 07^{\prime \prime} \mathrm{S}-79^{\circ} 36^{\prime} 22^{\prime \prime O}$ & $\mathrm{BSI}$ & $1600-2000$ \\
\hline & Distrito Sondor & $05^{\circ} 18^{\prime} 56^{\prime \prime} \mathrm{S}-79^{\circ} 24^{\prime} 36^{\prime \prime O}$ & BSI & $1700-2000$ \\
\hline & Distrito Carmen de la Frontera & $05^{\circ} 08^{\prime} 54^{\prime \prime} \mathrm{S}-79^{\circ} 25^{\prime} 41^{\prime \prime O}$ & $\mathrm{BHM}$ & $2450-3000$ \\
\hline \multirow[t]{7}{*}{ Ayabaca } & Ciudad de Ayabaca & $04^{\circ} 38^{\prime} 13^{\prime \prime} \mathrm{S}-79^{\circ} 43^{\prime} 26^{\prime \prime O}$ & $\mathrm{BHM}$ & $2715-3000$ \\
\hline & Distrito Jililí & $04^{\circ} 38^{\prime} 13^{\prime \prime} \mathrm{S}-79^{\circ} 43^{\prime} 26^{\prime \prime} \mathrm{O}$ & $\mathrm{BSI}$ & $1600-1900$ \\
\hline & Bosque de Cuyas & $04^{\circ} 36^{\prime} \mathrm{S}-79^{\circ} 42^{\prime} \mathrm{O}$ & $\mathrm{BHM}$ & $2100-2900$ \\
\hline & Caserío Socchabamba & $04^{\circ} 36^{\prime} 35^{\prime \prime} \mathrm{S}-79^{\circ} 42^{\prime} 34^{\prime \prime O}$ & $\mathrm{BHM}$ & $2300-3000$ \\
\hline & Distrito Suyo & $04^{\circ} 30^{\prime} 45^{\prime \prime} \mathrm{S}-80^{\circ} 00^{\prime} 10^{\prime \prime} \mathrm{O}$ & BESII/BESc & $200-800$ \\
\hline & Caserío Yacupampa & $05^{\circ} 10^{\prime} 51^{\prime \prime S}-80^{\circ} 37^{\prime} 31^{\prime \prime O}$ & $\mathrm{BHM}$ & $2700-3000$ \\
\hline & Simbaca & $04^{\circ} 72^{\prime} 98^{\prime \prime} \mathrm{S}-79^{\circ} 54^{\prime} 22^{\prime \prime} \mathrm{O}$ & $\mathrm{BHM}$ & 2000 \\
\hline
\end{tabular}

